\title{
Synthesis of Indolo[1,2-f]phenanthridines from Palladium-Catalyzed Reactions of Arynes
}

\author{
Chunsong Xie, Yuhong Zhang *, Zedu huang, Peixin Xu \\ Department of Chemistry, Zhejiang University, Hangzhou 310027, P.R. China. \\ E-mail: yhzhang@zjuem.zju.edu.cn
}

Supporting Information

\section{Content}

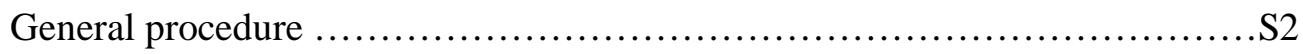

Characterization data of the starting materials $\ldots \ldots \ldots \ldots \ldots \ldots \ldots \ldots \ldots \ldots . . . \ldots \ldots$

Characterization data of the products................................. 14

UV-Vis absorbance spectra and fluorescence properties...................S32

References ......................................................... 35 
General All the reactions were carried out under the nitrogen atmosphere in oven-dried flasks. $\mathrm{CH}_{3} \mathrm{CN}$ was distilled from $\mathrm{CaH}_{2}$ using benzophenone as the indicator. Toluene and $\mathrm{THF}$ were distilled from $\mathrm{Na}$ using benzophenone as the indicator. O-(trimethylsilyl)phenyl triflate and 4,5-dimethyl-2-(trimethylsilyl)phenyl trifluoromethanesulfonate were prepared according to the method of reference 1. 1-(2-bromophenyl)indole and its derivatives were prepared according to the method of reference 2 . The other materials were purchased from common commercial sources and used without additional purification. ${ }^{1} \mathrm{H}$ NMR spectra were recorded at $400 \mathrm{MHz}$ using TMS as internal standard. ${ }^{13} \mathrm{C}$ NMR spectra were recorded at $100 \mathrm{MHz}$ using TMS as internal standard. Mass spectroscopy datas of the product were collected on an HRMS-ESI instrument.

Representative procedure for the preparation of 1-(2-bromophenyl)indole: Under $\mathrm{N}_{2}$, indole (1.17 g, $10 \mathrm{mmol})$, CuI (95 mg, $0.5 \mathrm{mmol})$, ethane-1,2-diamine (30 mg, $0.5 \mathrm{mmol})$, $\mathrm{K}_{3} \mathrm{PO}_{4}(4.24 \mathrm{~g}, 20 \mathrm{mmol})$ were added into a $50 \mathrm{~mL}$ oven-dried flask. A solution of 1-bromo-2-iodobenzene $(2.83 \mathrm{~g}, 10 \mathrm{mmol})$ in dioxane $(30 \mathrm{~mL})$ was added, the reaction mixture was allowed to reflux at $110^{\circ} \mathrm{C}$ for 24 hours. After the reaction completed and cooled to room temperature, the reaction mixture was filtered through a pad of cellite and the solvent was removed under reduced pressure. The residue was further purified by column chromatography on a silica gel column and the product was obtained as yellow oil. 
Representative procedure for the reaction of 1-(2-bromophenyl)indole with benzyne: 1-(2-bromophenyl)indole (136 mg, $0.5 \mathrm{mmol}), \mathrm{CsF}(228 \mathrm{mg}, 1.5 \mathrm{mmol}), \mathrm{Pd}_{2}(\mathrm{dba})_{3} \cdot \mathrm{CHCl}_{3}(13$ $\mathrm{mg}, 0.0125 \mathrm{mmol})$, dppp (10 mg, $0.025 \mathrm{mmol})$ were placed in an oven-dried flask. $\mathrm{CH}_{3} \mathrm{CN}(2$ $\mathrm{mL}$ ) and toluene $(2 \mathrm{~mL})$ were added under $\mathrm{N}_{2} . o$-(Trimethylsilyl)phenyl triflate (149 $\mathrm{mg}, 0.5$ $\mathrm{mmol})$ in $\mathrm{CH}_{3} \mathrm{CN}(1 \mathrm{~mL})$ and toluene $(1 \mathrm{~mL})$ was added dropwise. After the completion of the addition, the reaction mixture was allowed to react at $110{ }^{\circ} \mathrm{C}$ for $24 \mathrm{~h}$. Then the reaction mixture was cooled to room temperature and filtered through a pad of cellite. The filtrate was distilled under reduced pressure, and the product was obtained by flash chromatography on a silica gel column. 


\section{Characterization data of the starting materials}

(1) 1-(2-bromophenyl)-5-methoxy-1H-indole [ New compound ]

${ }^{1} \mathrm{H}$ NMR ( $400 \mathrm{MHz}, \mathrm{CDCl}_{3}$, TMS ) $\delta 7.74(\mathrm{~d}, J=8.4 \mathrm{~Hz}, 1 \mathrm{H}), 7.41(\mathrm{~m}, 2 \mathrm{H}), 7.29(\mathrm{~m}, 1 \mathrm{H})$, $7.20(\mathrm{~d}, J=3.2 \mathrm{~Hz}, 1 \mathrm{H}), 7.14(\mathrm{~d}, J=2.4 \mathrm{~Hz}, 1 \mathrm{H}), 6.98(\mathrm{~d}, J=8.8 \mathrm{~Hz}, 1 \mathrm{H}), 6.84$ ( d, $J=8.8$ $\mathrm{Hz}, 1 \mathrm{H}), 6.60\left(\mathrm{~d}, J=3.6 \mathrm{~Hz}, 1 \mathrm{H}\right.$ ), $3.86(\mathrm{~s}, 3 \mathrm{H}) ;{ }^{13} \mathrm{C} \mathrm{NMR}\left(100 \mathrm{MHz}, \mathrm{CDCl}_{3}\right) \delta 154.5$, 138.6, 133.9, 132.0, 129.6, 129.3, 129.2, 128.8, 128.2, 121.8, 112.4, 111.3, 102.8, 102.6, 55.8. HRMS (ESI) Calcd for $\mathrm{C}_{15} \mathrm{H}_{12} \mathrm{BrNO}$ : $[\mathrm{M}+\mathrm{H}]^{+}$302.0175; Found, 302.0185 .

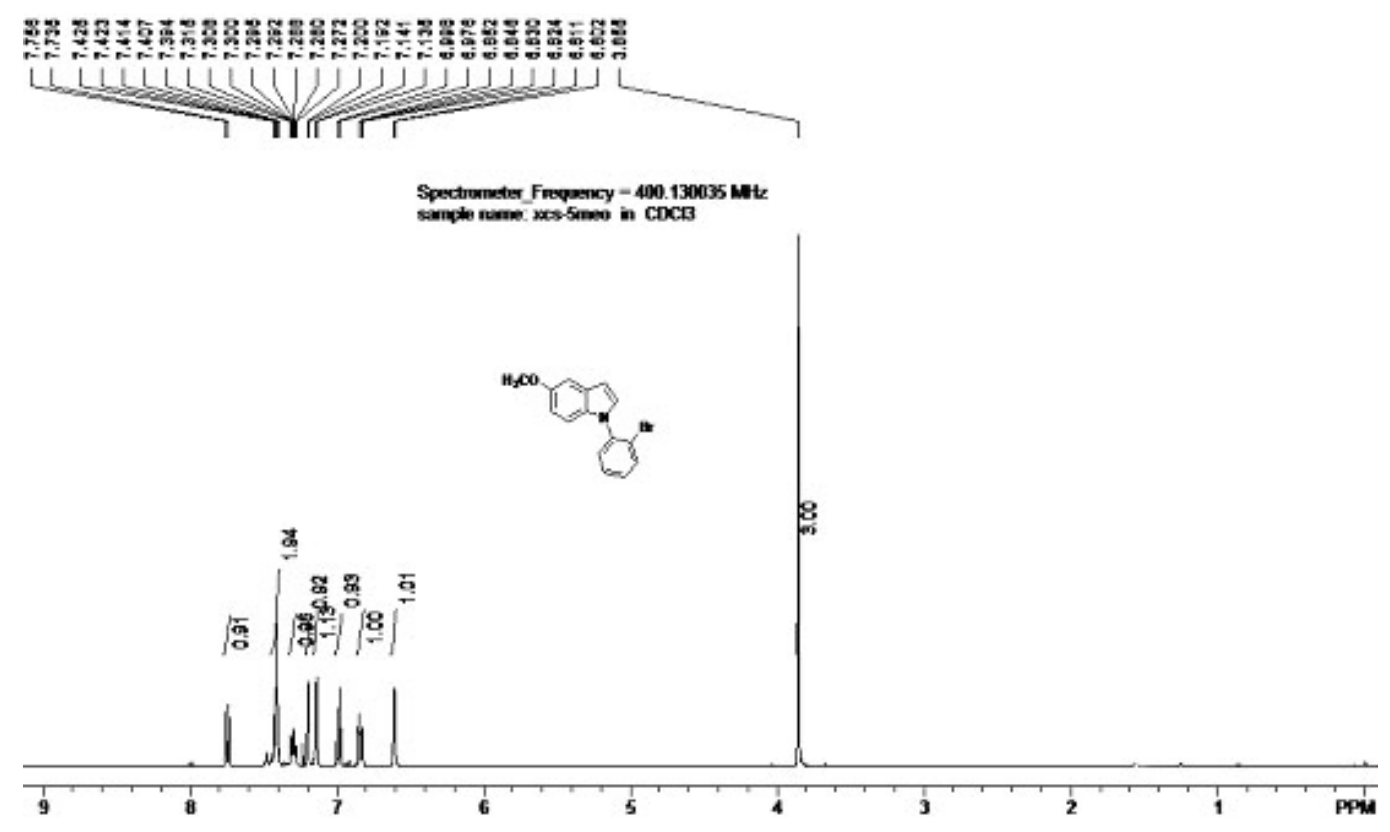




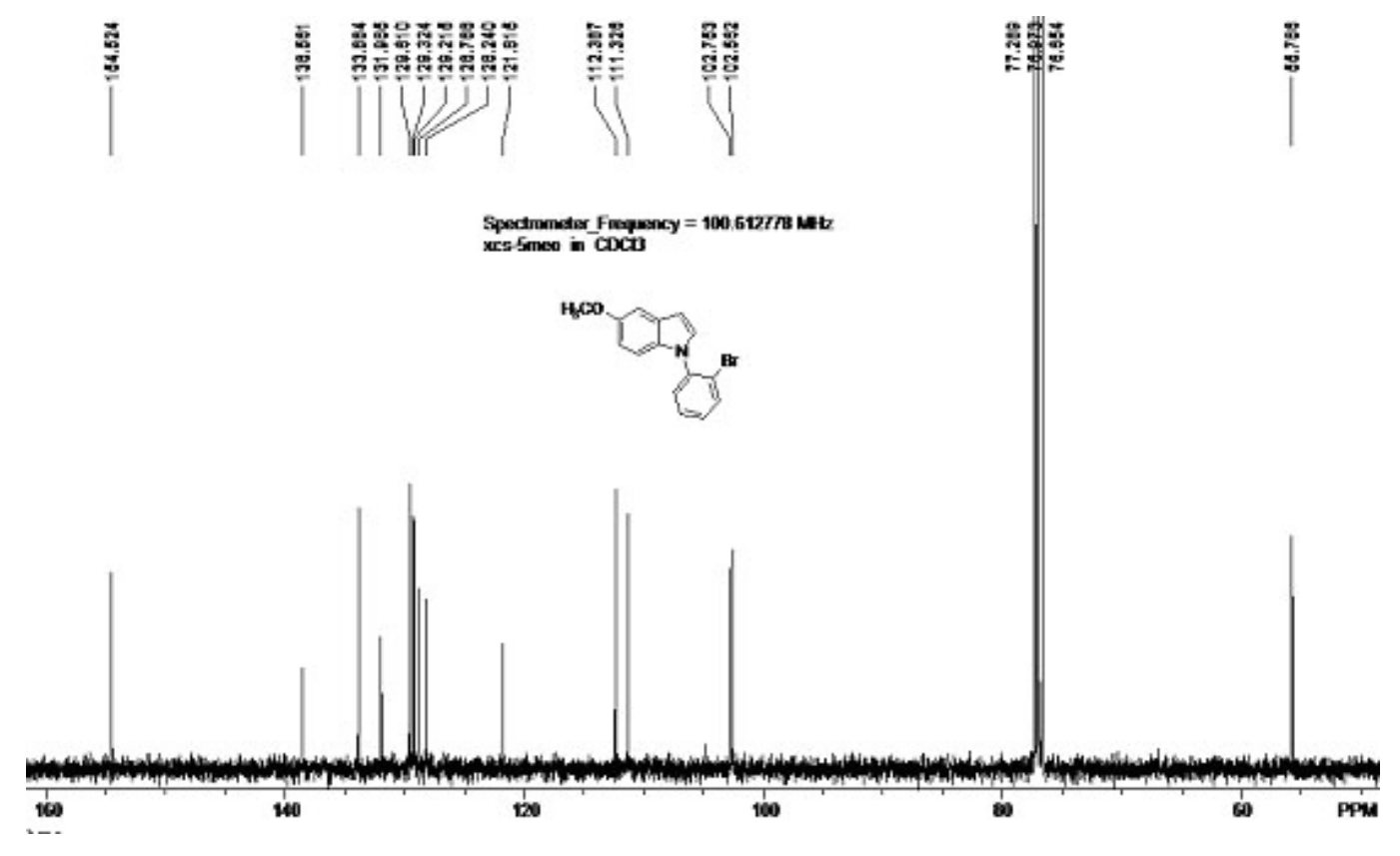

(2) 1-(2-bromophenyl)-6-methoxy-1H-indole [ New compound ]

${ }^{1} \mathrm{H}$ NMR ( $400 \mathrm{MHz}, \mathrm{CDCl}_{3}$, TMS ) $\delta 7.85(\mathrm{~d}, J=7.6 \mathrm{~Hz}, 1 \mathrm{H}$ ), $7.66(\mathrm{~d}, J=8.8 \mathrm{~Hz}, 2 \mathrm{H}$ ), $7.50(\mathrm{~d}, J=4.0 \mathrm{~Hz}, 1 \mathrm{H}), 7.37(\mathrm{~m}, 1 \mathrm{H}), 7.21(\mathrm{~d}, J=3.2 \mathrm{~Hz}, 1 \mathrm{H}), 6.96(\mathrm{~d}, J=9.2 \mathrm{~Hz}, 1 \mathrm{H})$, $6.73(\mathrm{~d}, J=3.2 \mathrm{~Hz}, 1 \mathrm{H}), 6.69(\mathrm{~d}, J=2 \mathrm{~Hz}, 1 \mathrm{H}), 3.85(\mathrm{~s}, 3 \mathrm{H}) ;{ }^{13} \mathrm{C}$ NMR ( $100 \mathrm{MHz}$, $\left.\mathrm{CDCl}_{3}\right) \delta 156.8,138.6,137.6,134.0,129.8,129.6,129.5,128.5,127.7,122.6,122.0,121.6$, 110.3, 103.1, 94.3, 55.7. HRMS (ESI) Calcd for $\mathrm{C}_{15} \mathrm{H}_{12} \mathrm{BrNO}$ : $[\mathrm{M}+\mathrm{H}]^{+}$302.0175; Found, 302.0185 . 


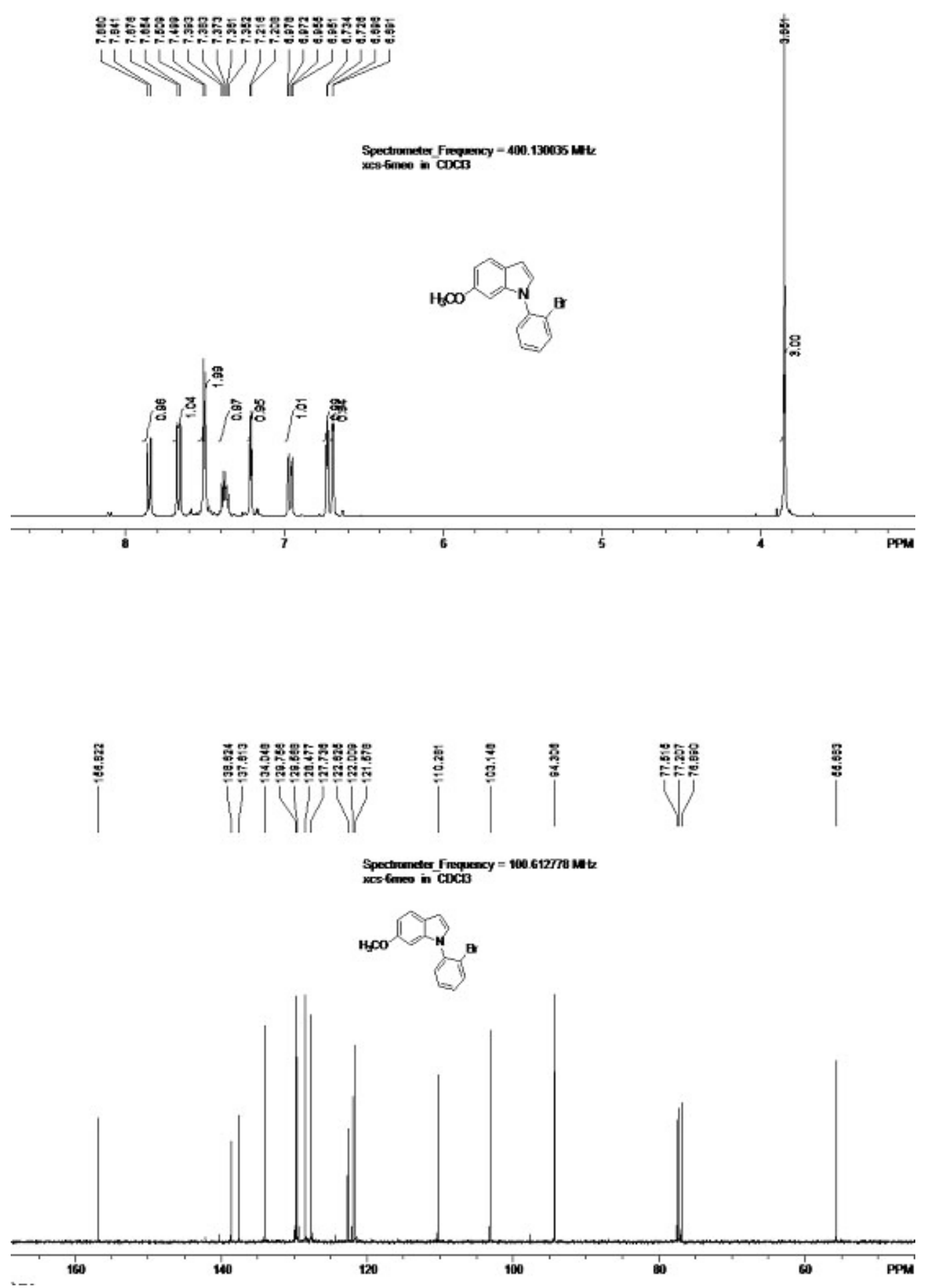


(3) 1-(2-bromophenyl)-5-methyl-1H-indole [ New compound ]

${ }^{1} \mathrm{H}$ NMR ( $400 \mathrm{MHz}, \mathrm{CDCl}_{3}$, TMS ) $\delta 7.84(\mathrm{~d}, J=8 \mathrm{~Hz}, 1 \mathrm{H}$ ), $7.58(\mathrm{~s}, 1 \mathrm{H}$ ), $7.50(\mathrm{~s}, 1 \mathrm{H})$, $7.49(\mathrm{~s}, 1 \mathrm{H}), 7.37(\mathrm{~m}, 1 \mathrm{H}), 7.28(\mathrm{~d}, J=3.2 \mathrm{~Hz}, 1 \mathrm{H}), 7.11(\mathrm{~m}, 2 \mathrm{H}), 6.71(\mathrm{~d}, J=2.8 \mathrm{~Hz}, 1$ $\mathrm{H}$ ), 2.57 ( s, $3 \mathrm{H}$ ); ${ }^{13} \mathrm{C}$ NMR ( $100 \mathrm{MHz}, \mathrm{CDCl}_{3}$ ) $\delta 138.7,135.2,133.9,129.7,129.6,129.4$, 128.8, 128.7, 128.3, 123.9, 121.9, 120.7, 110.3, 102.7, 21.5. HRMS (ESI) Calcd for $\mathrm{C}_{15} \mathrm{H}_{12} \mathrm{BrN}$ : $[\mathrm{M}+\mathrm{H}]^{+}$286.0226; Found, 286.0240.

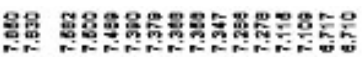

UMUVJjJ
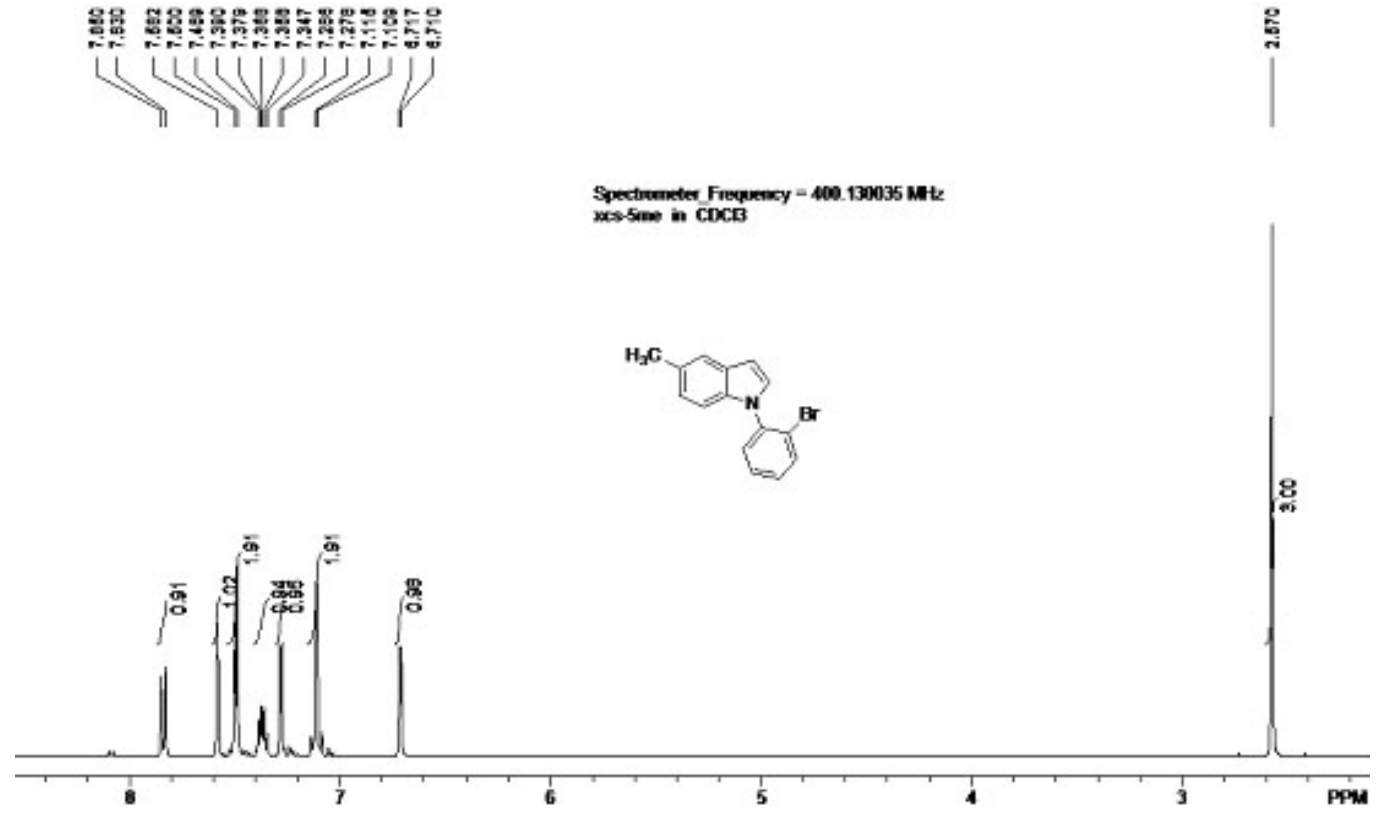


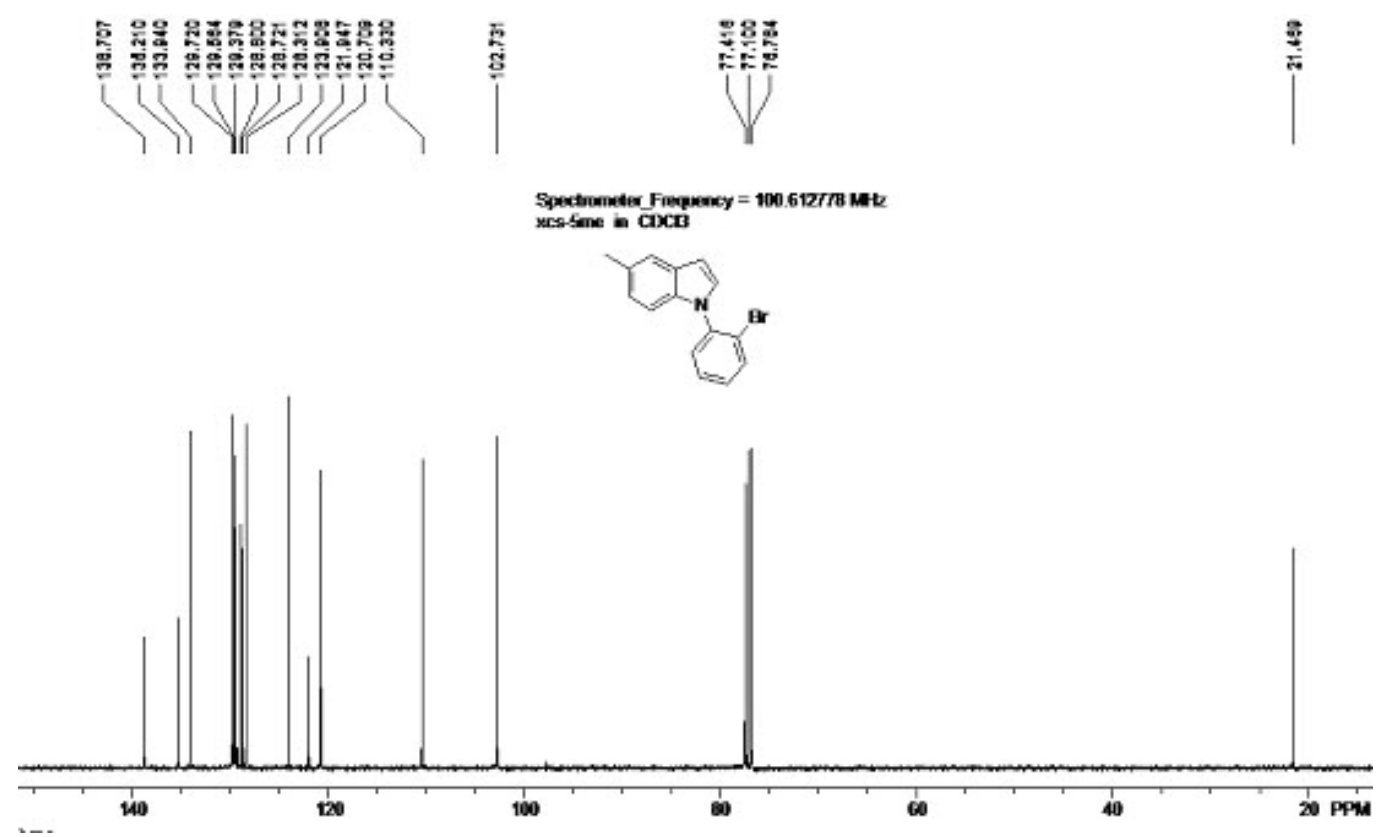

(4) 1-(2-bromophenyl)-4-methyl-1H-indole [ New compound ]

${ }^{1} \mathrm{H}$ NMR ( $400 \mathrm{MHz}, \mathrm{CDCl}_{3}$, TMS ) $\delta 7.86(\mathrm{~d}, J=8 \mathrm{~Hz}, 1 \mathrm{H}), 7.52(\mathrm{~m}, 2 \mathrm{H}), 7.39(\mathrm{~m}, 1 \mathrm{H})$, $7.34(\mathrm{~d}, J=3.2 \mathrm{~Hz}, 1 \mathrm{H}$ ), 7.23 ( t $, J=7.6 \mathrm{~Hz}, 1 \mathrm{H}$ ), 7.09 ( q, $2 \mathrm{H}$ ), $6.84(\mathrm{~d}, J=3.2 \mathrm{~Hz}, 1 \mathrm{H})$, 2.74 ( s, $3 \mathrm{H}$ ); ${ }^{13} \mathrm{C}$ NMR ( $100 \mathrm{MHz}, \mathrm{CDCl}_{3}$ ) $\delta 138.7$, 136.6, 134.0, 130.5, 129.8, 129.5, 128.3, 128.2, 128.1, 122.5, 120.6, 108.3, 101.7, 18.8. HRMS (ESI) Calcd for $\mathrm{C}_{15} \mathrm{H}_{12} \mathrm{BrN}:[\mathrm{M}+\mathrm{H}]^{+}$ 286.0226; Found, 286.0240. 


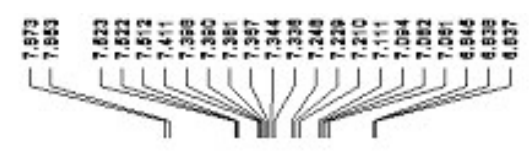

\section{Spectrouder Fropency $=400.130035 \mathrm{MHE}$ yes-1me in COCB}
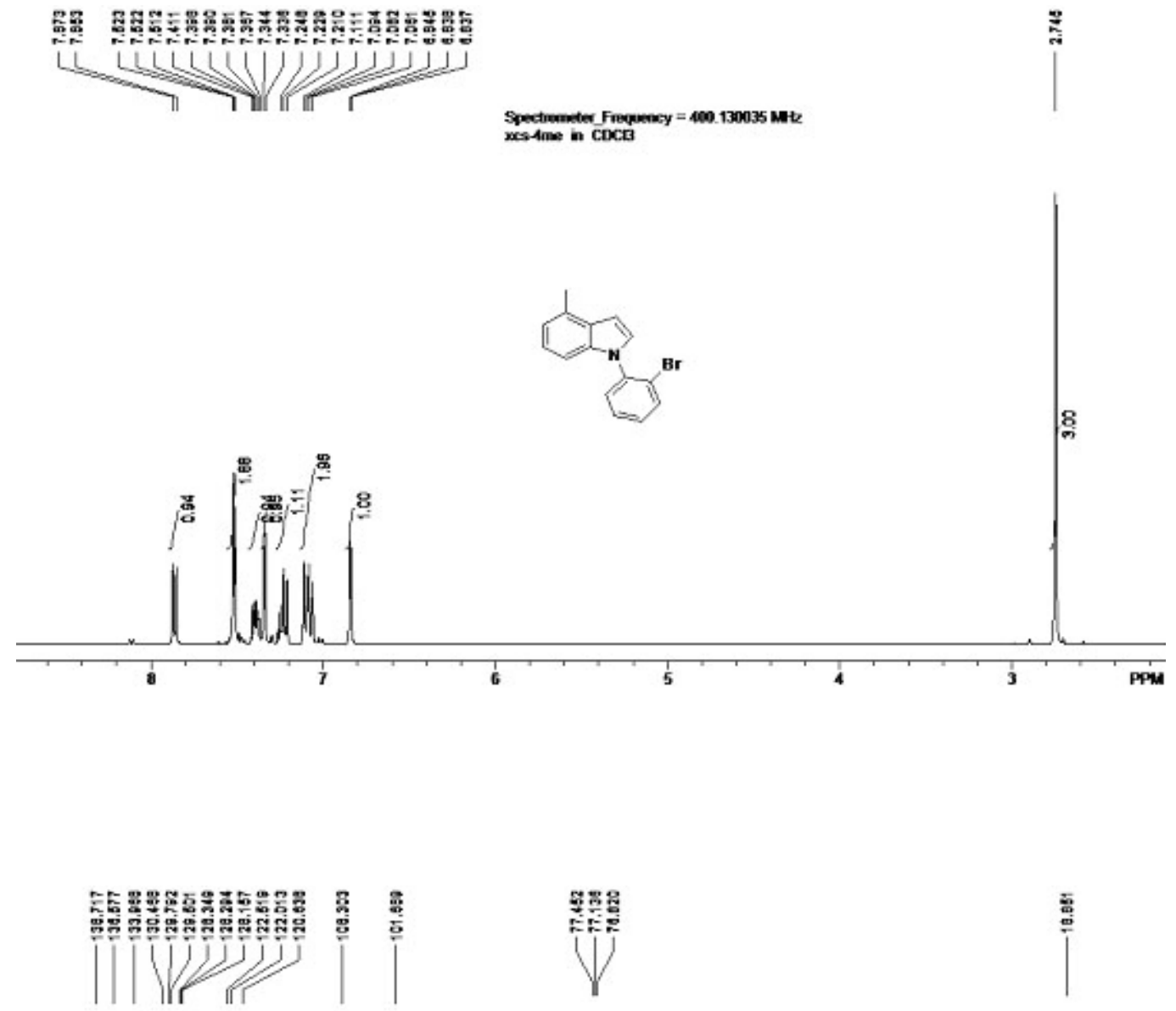

Spectrometer Frequency $=100.6127 \mathrm{rs}$ itt xess Ame in $\overline{\mathrm{CDCCB}}$

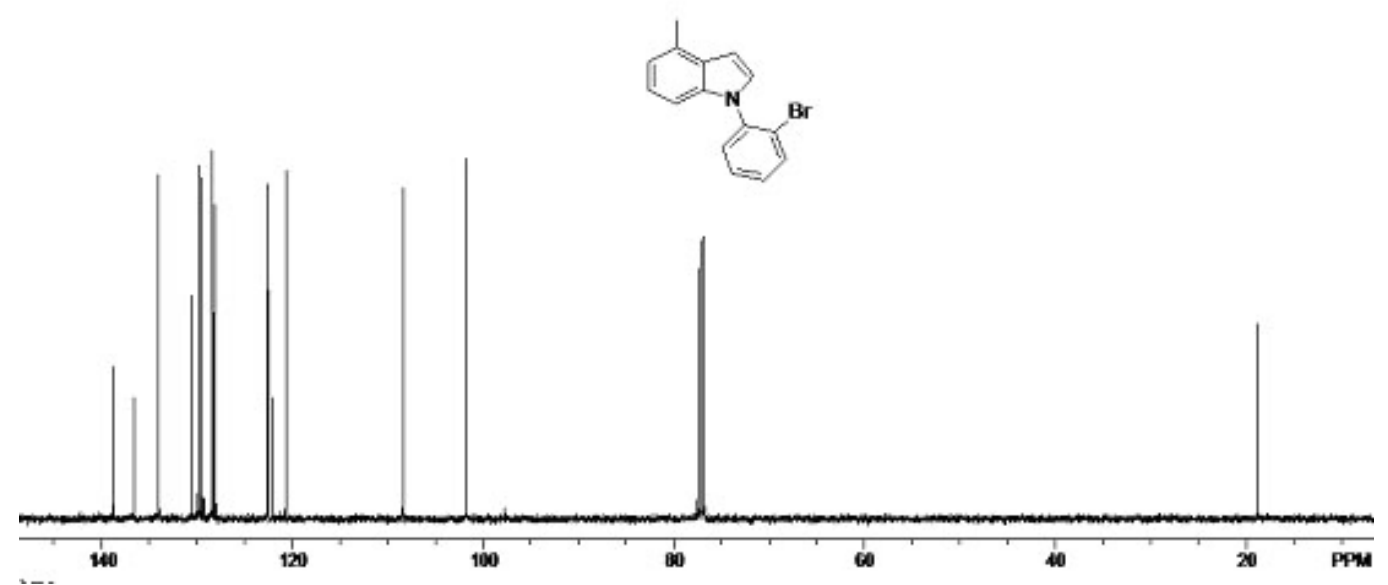


(5) 1-(2-bromophenyl)-1H-indole [ New compound ]

${ }^{1} \mathrm{H}$ NMR ( $400 \mathrm{MHz}, \mathrm{CDCl}_{3}$, TMS ) $\delta 7.85$ ( m, $2 \mathrm{H}$ ), $7.52(\mathrm{~m}, 2 \mathrm{H}$ ), 7.40 ( m, $1 \mathrm{H}$ ), 7.36 ( d, $J=3.6 \mathrm{~Hz}, 1 \mathrm{H}), 7.33(\mathrm{~m}, 2 \mathrm{H}), 7.25(\mathrm{~d}, J=8.4 \mathrm{~Hz}, 1 \mathrm{H}), 6.84(\mathrm{~d}, J=3.6 \mathrm{~Hz}, 1 \mathrm{H}) ;{ }^{13} \mathrm{C}$ NMR ( $\left.100 \mathrm{MHz}, \mathrm{CDCl}_{3}\right) \delta 138.6,136.8,134.0,129.8,129.6,128.8,128.5,128.4,122.4$, 122.0, 121.1, 120.4, 110.7, 103.3. HRMS (ESI) Calcd for $\mathrm{C}_{14} \mathrm{H}_{10} \mathrm{BrN}$ : $[\mathrm{M}+\mathrm{H}]^{+} 272.0069$; Found, 272.0080.

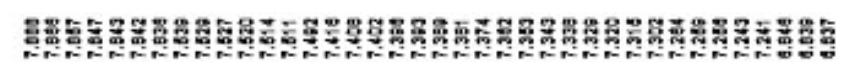

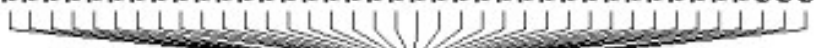

7 Tiir $r$

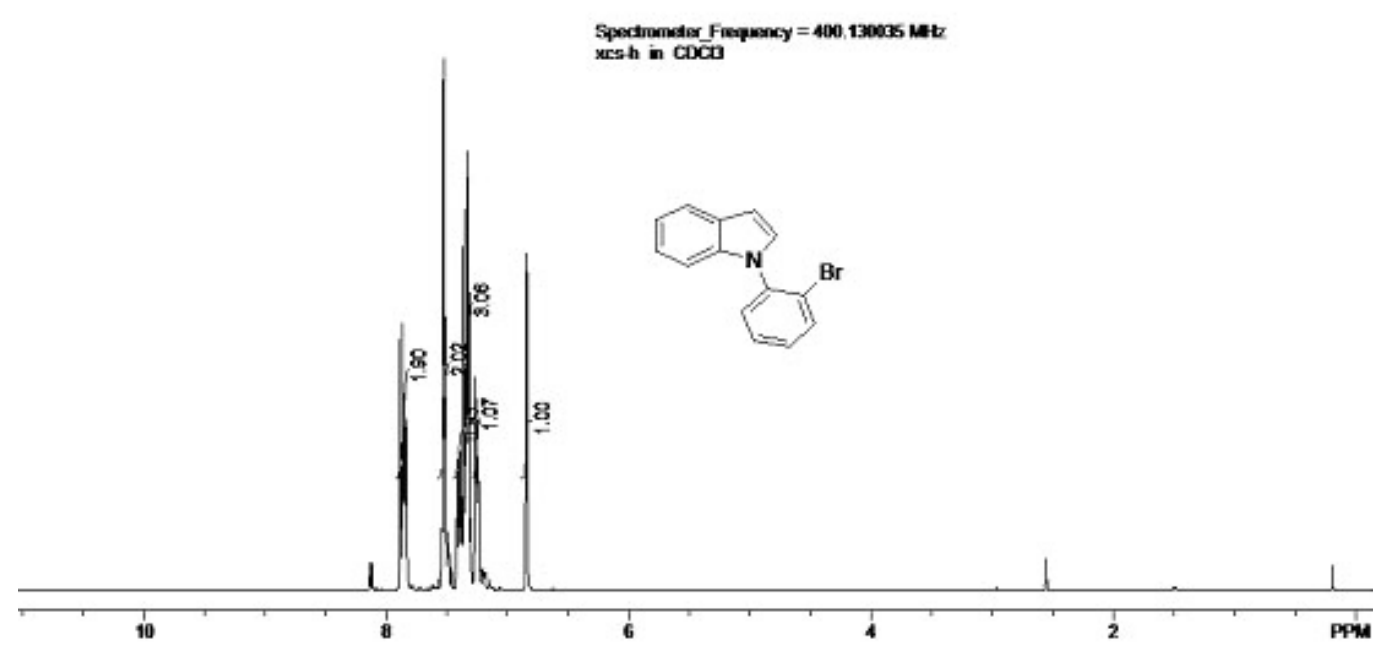



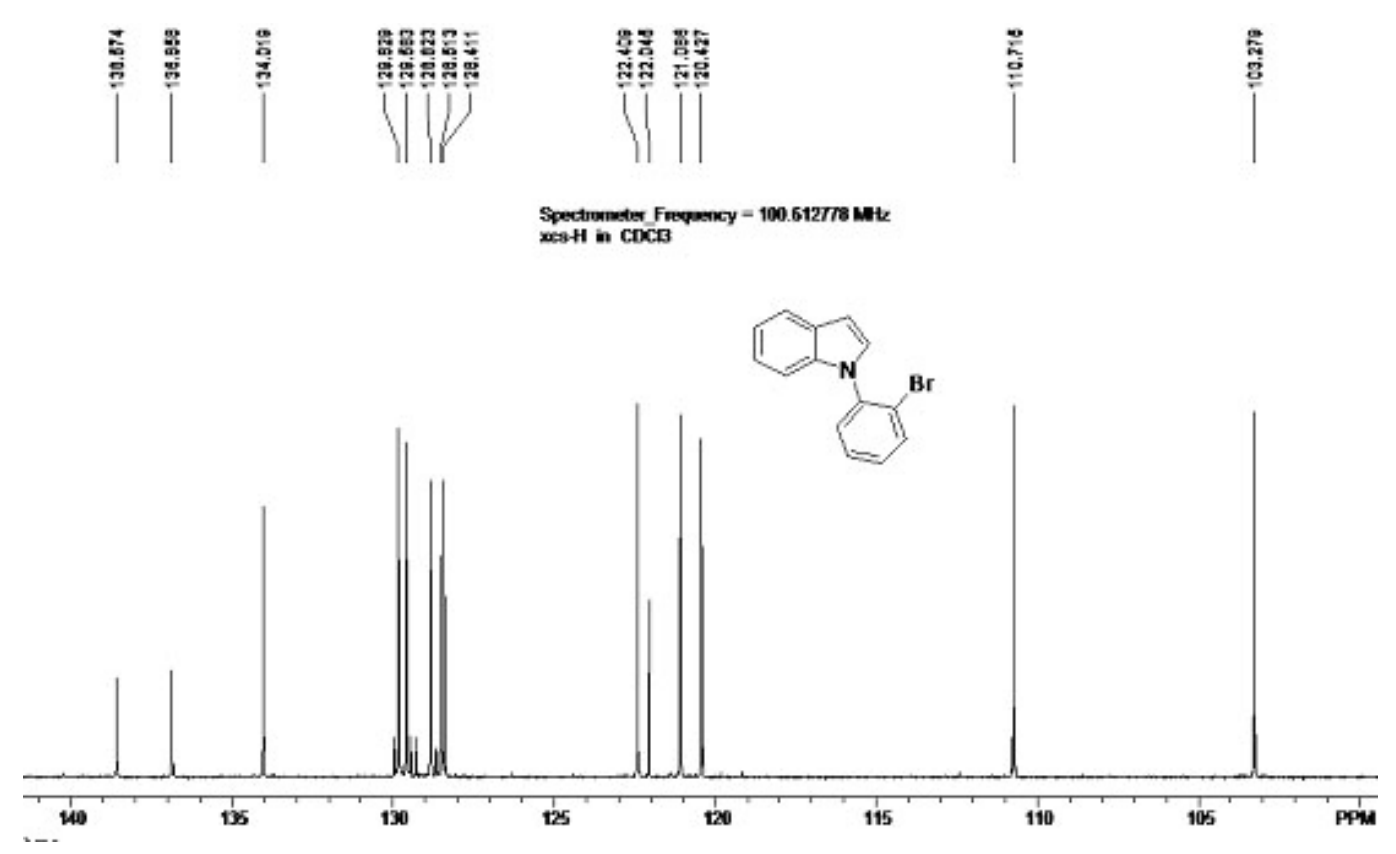

(6) methyl 1-(2-bromophenyl)-1H-indole-4-carboxylate [ New compound ]

${ }^{1} \mathrm{H}$ NMR ( $400 \mathrm{MHz}, \mathrm{CDCl}_{3}$, TMS ) $\delta 7.96(\mathrm{~d}, J=6.8 \mathrm{~Hz}, 1 \mathrm{H}), 7.73(\mathrm{~d}, J=8.4 \mathrm{~Hz}, 1 \mathrm{H}$ ), $7.55(\mathrm{t}, J=7.8 \mathrm{~Hz}, 2 \mathrm{H}), 7.50(\mathrm{~d}, J=3.2 \mathrm{~Hz}, 1 \mathrm{H}), 7.47(\mathrm{~d}, J=3.2 \mathrm{~Hz}, 1 \mathrm{H}), 7.41(\mathrm{t}, J=7.4$ $\mathrm{Hz}, 1 \mathrm{H}$ ), 7.34 ( d, $J=2.8 \mathrm{~Hz}, 1 \mathrm{H}$ ), 7.26 ( t $J=8.0 \mathrm{~Hz}, 1 \mathrm{H}$ ), $4.02(\mathrm{~s}, 3 \mathrm{H}) ;{ }^{13} \mathrm{C} \mathrm{NMR}(100$ $\left.\mathrm{MHz}, \mathrm{CDCl}_{3}\right) \delta 167.8,140.2,137.5,133.9,130.8,129.9,129.8,128.5,128.0,123.8,121.8$, 121.6, 115.6, 115.5, 104.3, 51.8. HRMS (ESI) Calcd for $\mathrm{C}_{16} \mathrm{H}_{12} \mathrm{BrNO}_{2}$ : $[\mathrm{M}+\mathrm{H}]^{+} 330.0124$; Found, 330.0126. 

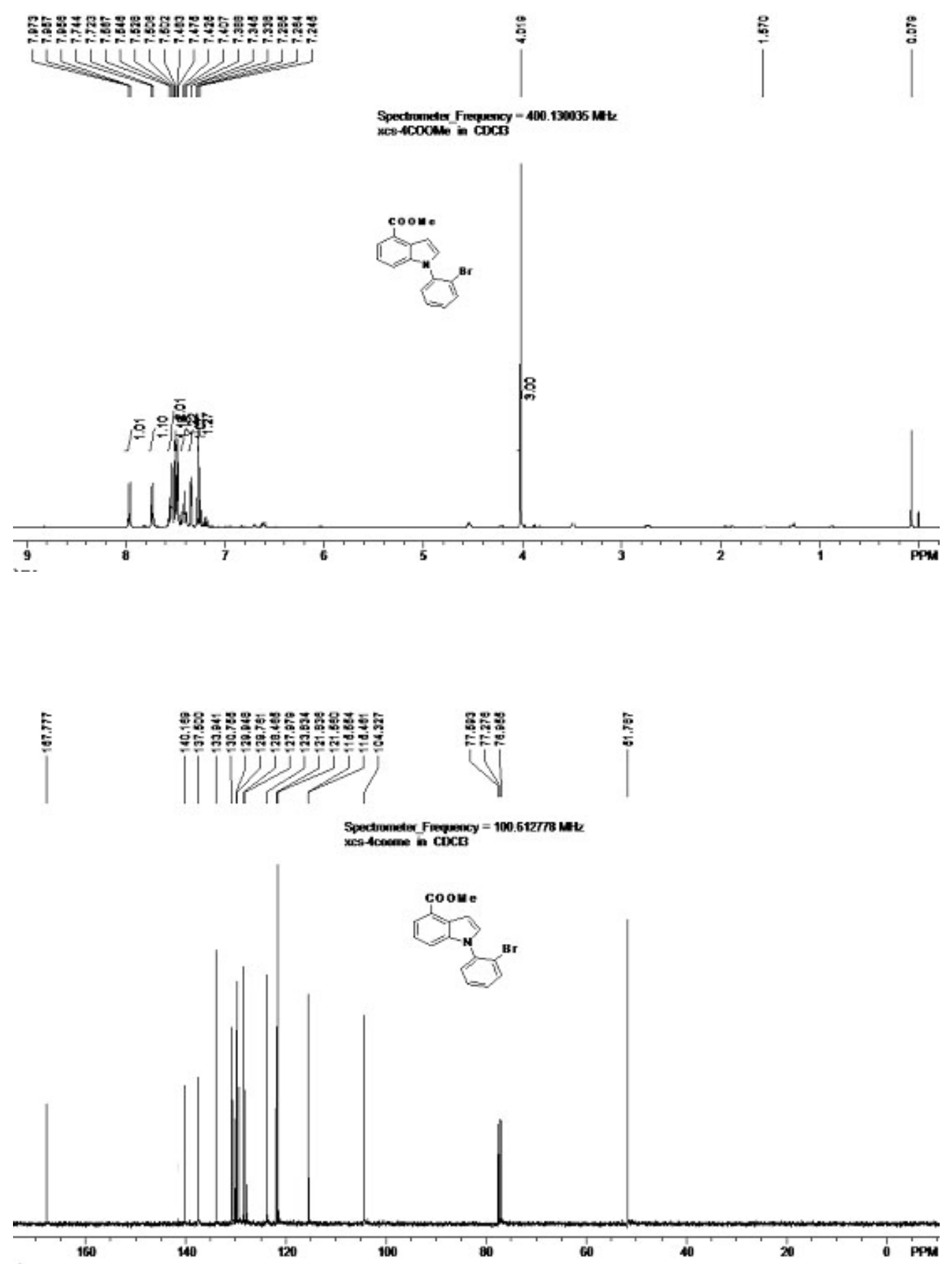
(7) 1-(2-bromophenyl)-1H-indole-5-carbonitrile [ New compound ]

${ }^{1} \mathrm{H}$ NMR ( $400 \mathrm{MHz}, \mathrm{CDCl}_{3}$, TMS ) $\delta 7.80(\mathrm{~d}, J=8.0 \mathrm{~Hz}, 1 \mathrm{H}$ ), $7.52(\mathrm{~m}, 2 \mathrm{H}), 7.37$ ( d, $J=$ $8.0 \mathrm{~Hz}, 1 \mathrm{H}$ ), 7.33 ( d, $J=3.2 \mathrm{~Hz}, 1 \mathrm{H}$ ), 7.30 ( d, $J=3.2 \mathrm{~Hz}, 1 \mathrm{H}$ ), 7.24 ( t, $J=7.8 \mathrm{~Hz}, 1 \mathrm{H}$ ), $7.12(\mathrm{~d}, J=8.4 \mathrm{~Hz}, 1 \mathrm{H}), 7.07(\mathrm{~d}, J=8.4 \mathrm{~Hz}, 1 \mathrm{H}) ;{ }^{13} \mathrm{C} \mathrm{NMR}\left(100 \mathrm{MHz}, \mathrm{CDCl}_{3}\right) \delta 140.3$, 134.0, 131.0, 132.8, 130.6, 129.6, 129.2, 128.6, 128.1, 126.5, 125.2, 121.9, 120.5, 111.5, 103.8. HRMS (ESI) Calcd for $\mathrm{C}_{15} \mathrm{H}_{9} \mathrm{BrN}_{2}$ : [M+Na] $]^{+}$318.9841; Found, 318.9850.
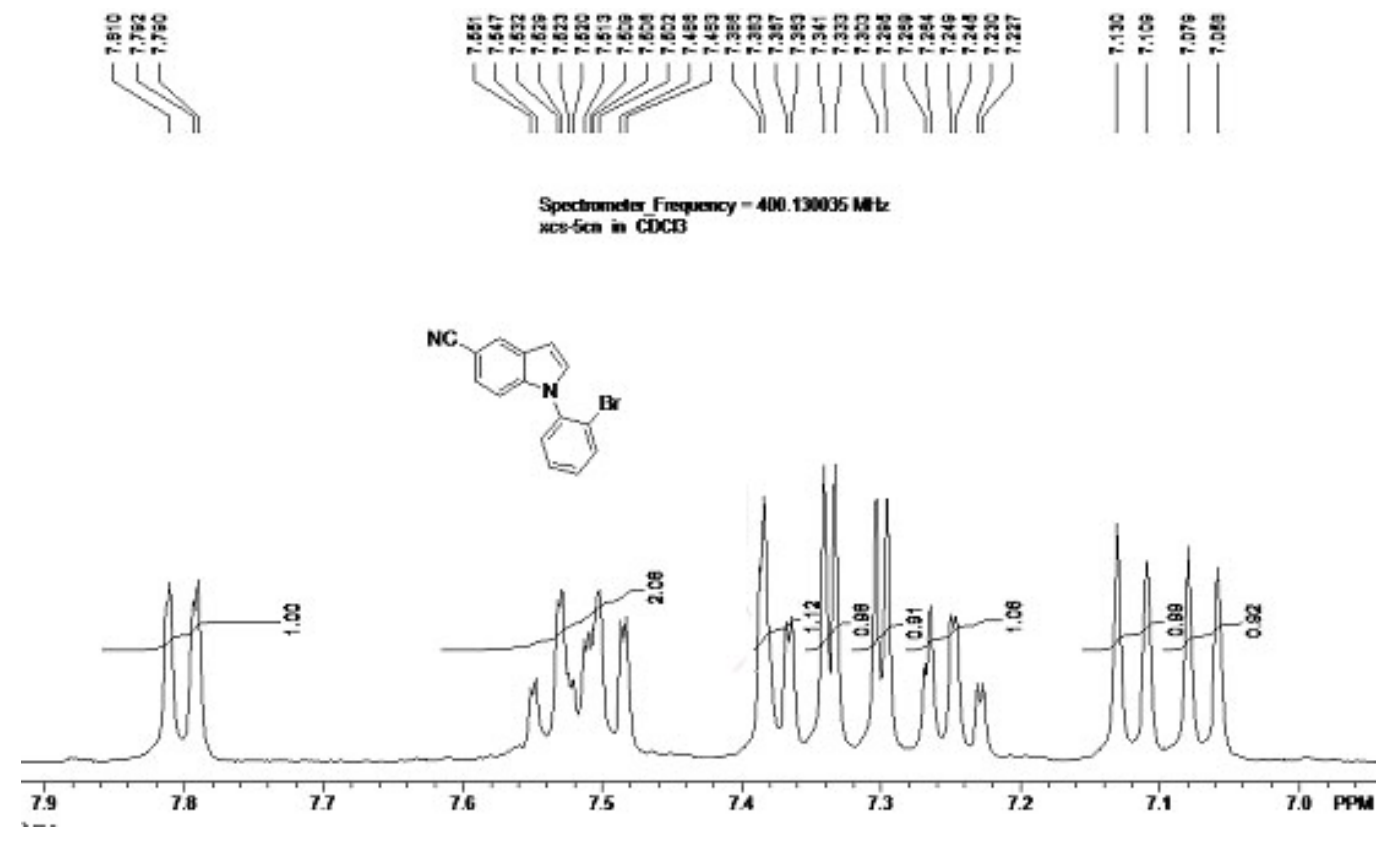


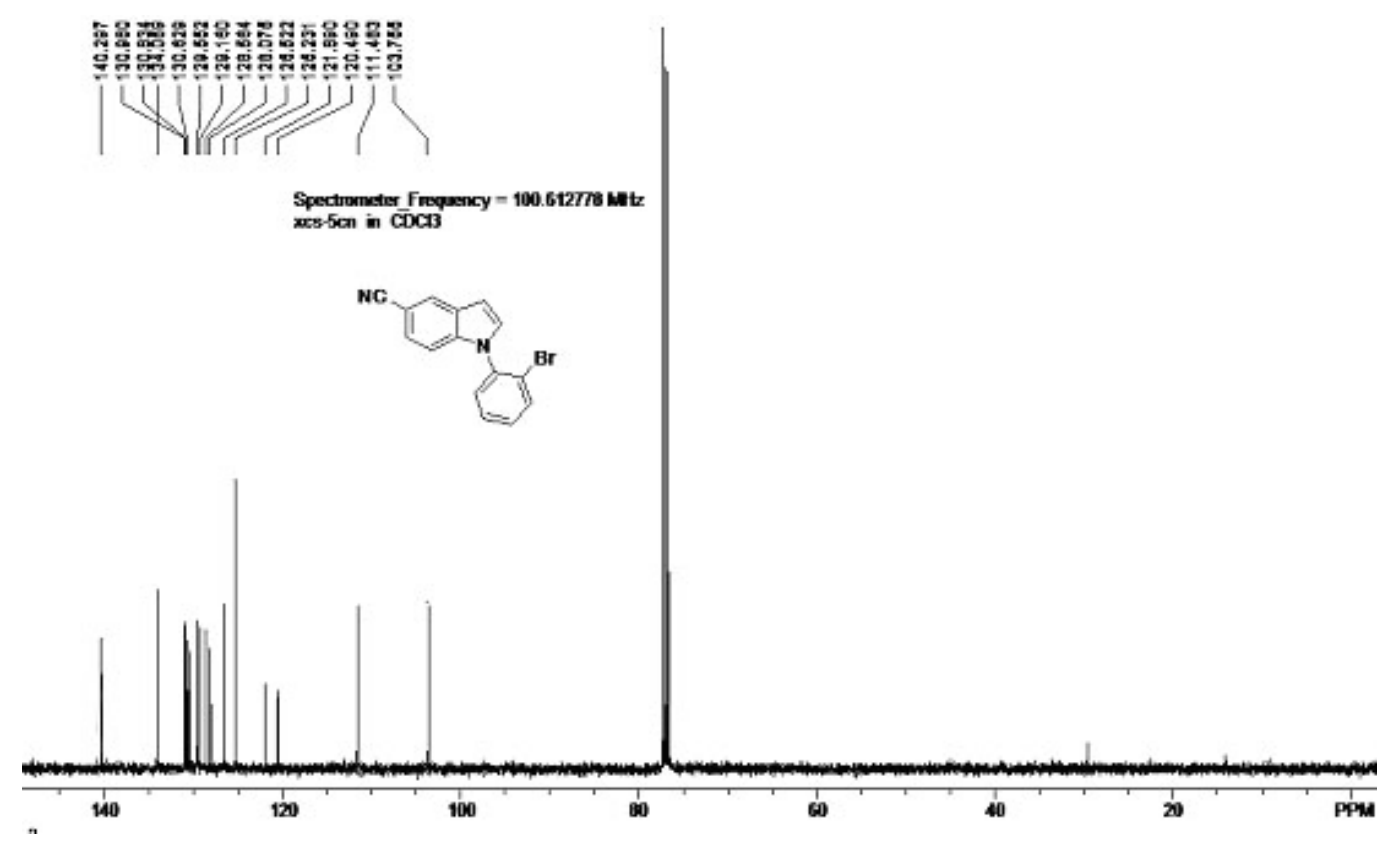

\section{Characterization data of the products}

(1) 11-methoxyindolo[1,2-f]phenanthridine [ T 2-1, New compound ]

${ }^{1} \mathrm{H}$ NMR ( $400 \mathrm{MHz}, \mathrm{CDCl}_{3}$, TMS ) $\delta 8.48(\mathrm{~d}, J=8.4 \mathrm{~Hz}, 1 \mathrm{H}$ ), 8.34 ( d, $J=8 \mathrm{~Hz}, 1 \mathrm{H}$ ), 8.26 $(\mathrm{m}, 2 \mathrm{H}), 8.13(\mathrm{~m}, 1 \mathrm{H}), 7.59(\mathrm{t}, J=7.6 \mathrm{~Hz}, 1 \mathrm{H}), 7.52(\mathrm{~m}, 2 \mathrm{H}), 7.36(\mathrm{t}, J=7.6 \mathrm{~Hz}, 1 \mathrm{H})$, 7.27 ( s, $1 \mathrm{H}$ ), 7.20 ( s, $1 \mathrm{H}$ ), 7.04 ( d, $J=9.2 \mathrm{~Hz}, 1 \mathrm{H}$ ), 3.96 ( s, $3 \mathrm{H}$ ); ${ }^{13} \mathrm{C}$ NMR ( $100 \mathrm{MHz}$, $\left.\mathrm{CDCl}_{3}\right) \delta 154.9,137.0,136.6,135.9,135.4,131.4,128.9,127.9,124.5,124.4,123.6,123.5$, 122.8, 122.4, 121.8, 115.6, 114.9, 111.2, 101.9, 94.8, 55.5. HRMS (ESI) Calcd for $\mathrm{C}_{21} \mathrm{H}_{15} \mathrm{NO}$ : $[\mathrm{M}+\mathrm{H}]^{+}$298.1222; Found, 298.1222. Yellow powder, mp 155-156 ${ }^{\circ} \mathrm{C}$. 

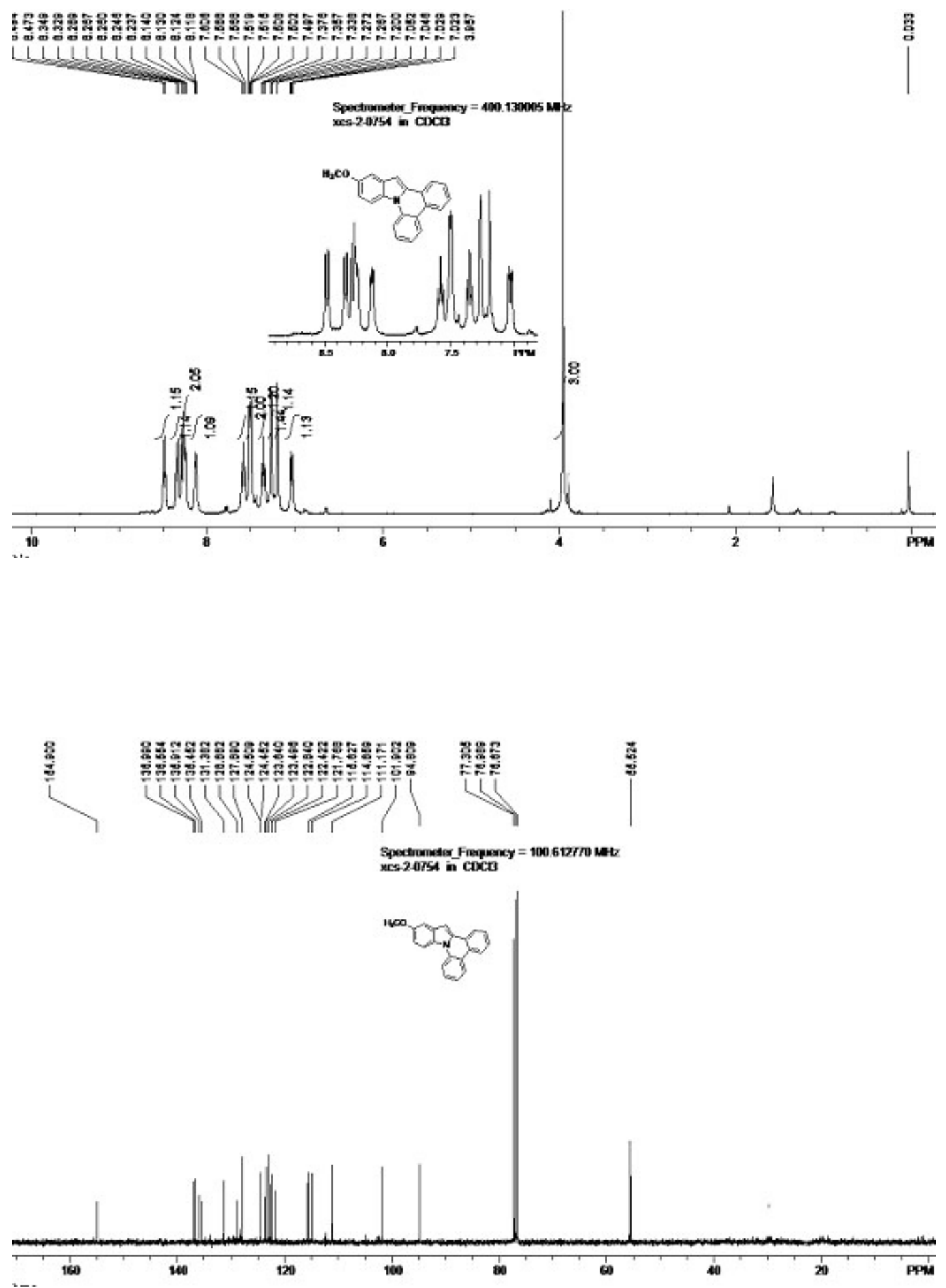
(2) 12-methoxyindolo[1,2-f]phenanthridine [ T 2-2, New compound ]

${ }^{1} \mathrm{H}$ NMR ( $400 \mathrm{MHz}, \mathrm{CDCl}_{3}$, TMS ) $\delta 8.48(\mathrm{~d}, J=9.6 \mathrm{~Hz}, 1 \mathrm{H}$ ), $8.33(\mathrm{~d}, J=8 \mathrm{~Hz}, 1 \mathrm{H}$ ), 8.22 $(\mathrm{m}, 1 \mathrm{H}), 8.10(\mathrm{~m}, 1 \mathrm{H}), 7.90(\mathrm{~d}, J=2.0 \mathrm{~Hz}, 1 \mathrm{H}), 7.74(\mathrm{~d}, J=8.4 \mathrm{~Hz}, 1 \mathrm{H}), 7.59(\mathrm{t}, J=7.8$ $\mathrm{Hz}, 1 \mathrm{H}$ ), 7.49 ( m, $2 \mathrm{H}$ ), 7.37 ( t, $J=7.8 \mathrm{~Hz}, 1 \mathrm{H}$ ), 7.27 ( s, $1 \mathrm{H}$ ), 7.06 ( d, J=4.2 Hz, $1 \mathrm{H}$ ), 4.00 ( s, $3 \mathrm{H}$ ); ${ }^{13} \mathrm{C}$ NMR ( $\left.100 \mathrm{MHz}, \mathrm{CDCl}_{3}\right) \delta 156.1,135.9$, 134.5, 134.3, 128.5, 128.1, 127.3, $126.45,126.37,124.6,123.9,123.6,122.9,122.3,122.2,121.3,115.9,110.8,99.0,95.9,56.0$. HRMS (ESI) Calcd for $\mathrm{C}_{21} \mathrm{H}_{15} \mathrm{NO}$ : $[\mathrm{M}+\mathrm{H}]^{+}$298.1222; Found, 298.1222. Yellow powder, mp $184-185^{\circ} \mathrm{C}$.

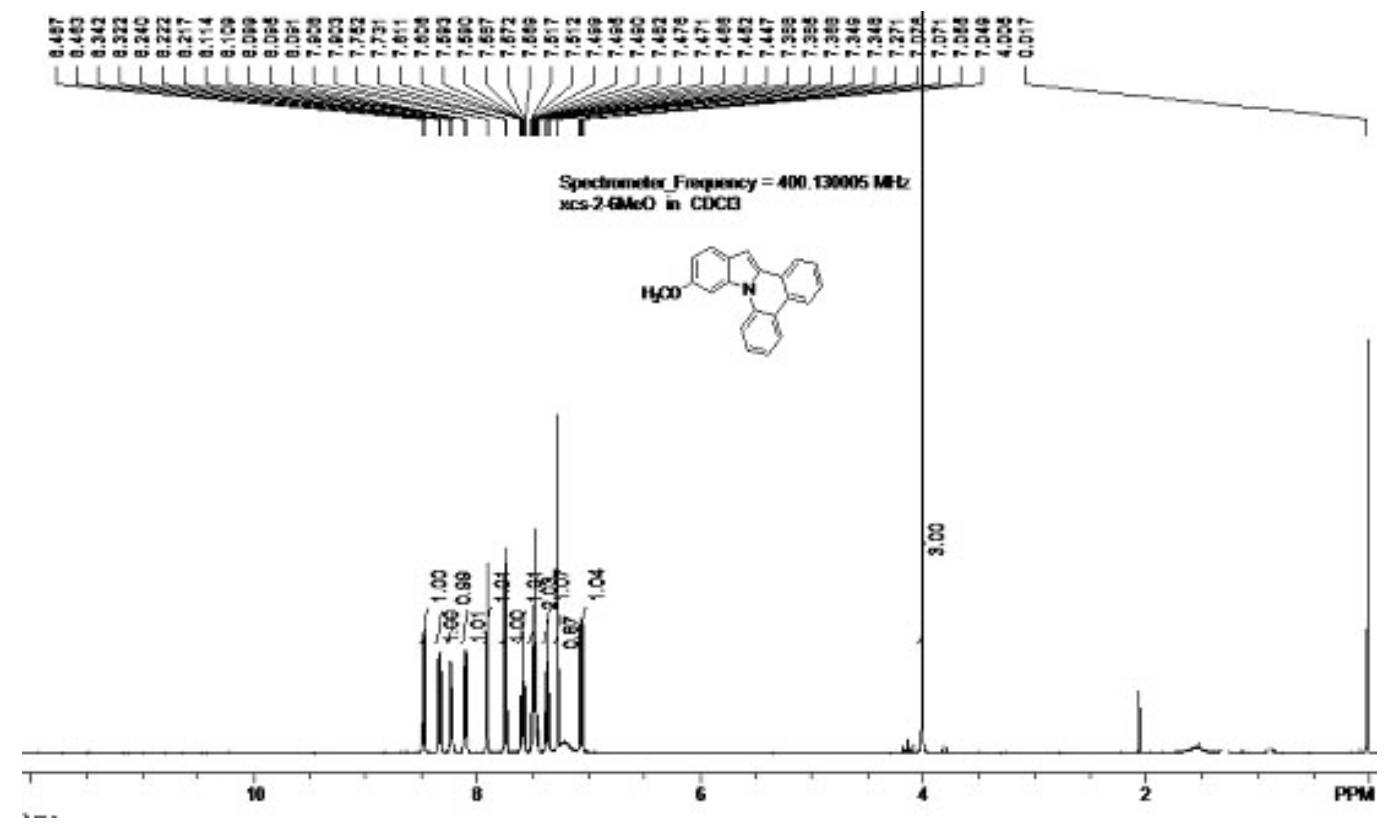




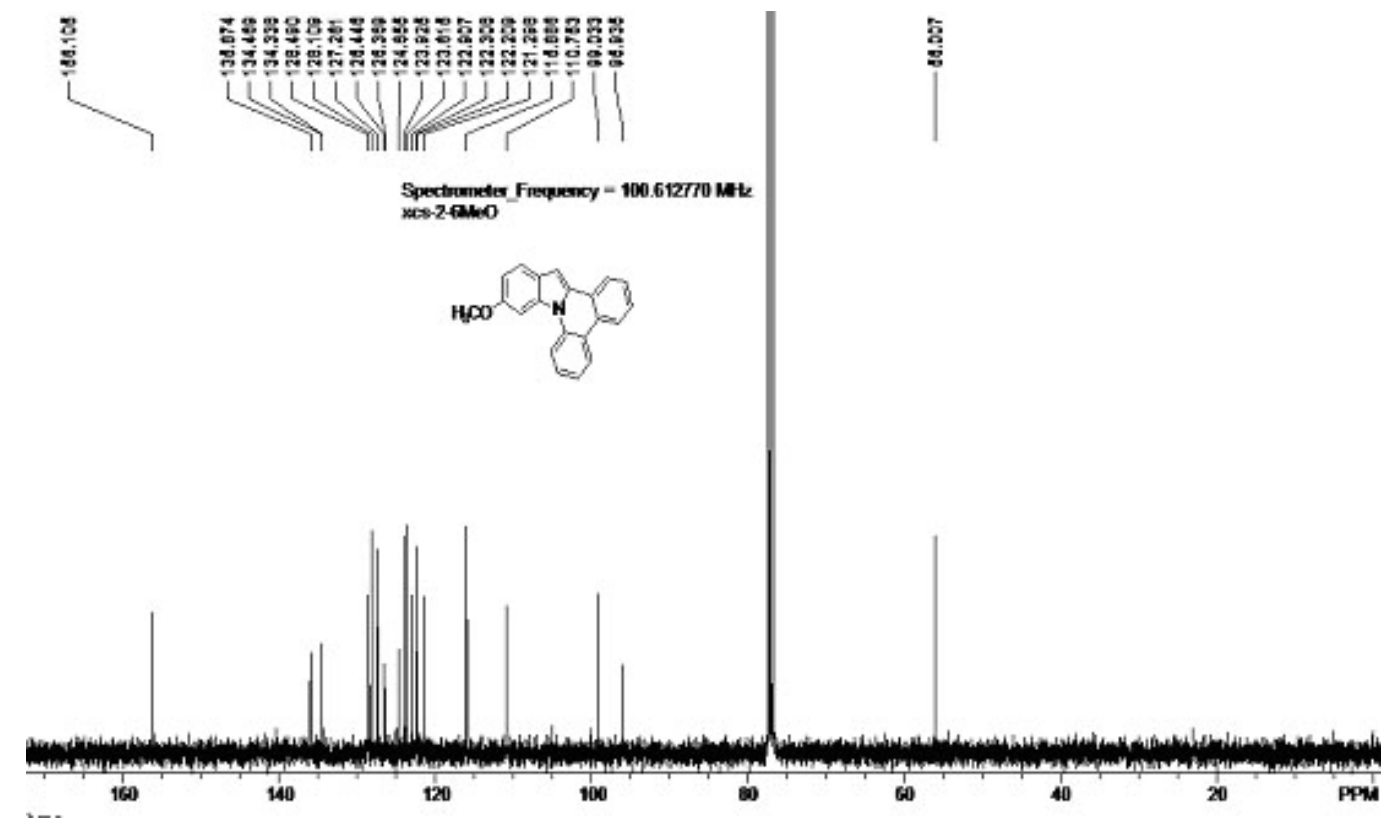

(3) 11-methylindolo[1,2-f]phenanthridine [ T 2-3, New compound ]

${ }^{1} \mathrm{H}$ NMR ( $400 \mathrm{MHz}, \mathrm{CDCl}_{3}$, TMS ) $\delta 8.52(\mathrm{~d}, J=8.4 \mathrm{~Hz}, 1 \mathrm{H}$ ), $8.33(\mathrm{~d}, J=8 \mathrm{~Hz}, 1 \mathrm{H}$ ), 8.25 $(\mathrm{m}, 2 \mathrm{H}), 8.13(\mathrm{~m}, 1 \mathrm{H}), 7.58(\mathrm{~m}, 2 \mathrm{H}), 7.49(\mathrm{t}, J=4.6 \mathrm{~Hz}, 2 \mathrm{H}), 7.35$ ( t $, J=7.6 \mathrm{~Hz}, 1 \mathrm{H})$, $7.21\left(\mathrm{~m}, 2 \mathrm{H}\right.$ ), 2.54 ( s, $3 \mathrm{H}$ ); ${ }^{13} \mathrm{C} \mathrm{NMR}\left(100 \mathrm{MHz}, \mathrm{CDCl}_{3}\right) \delta$ 136.0, 135.2, 132.2, 131.2, 130.6, 128.7, 128.1, 127.7, 126.8, 126.2, 124.1, 123.9, 123.6, 122.8, 122.4, 121.9, 120.6, 116.1, 113.9, 95.7, 21.4. HRMS (ESI) Calcd for $\mathrm{C}_{21} \mathrm{H}_{15} \mathrm{~N}$ : $[\mathrm{M}+\mathrm{H}]^{+}$282.1277; Found, 282.1273. Yellow powder, mp $178-180^{\circ} \mathrm{C}$. 

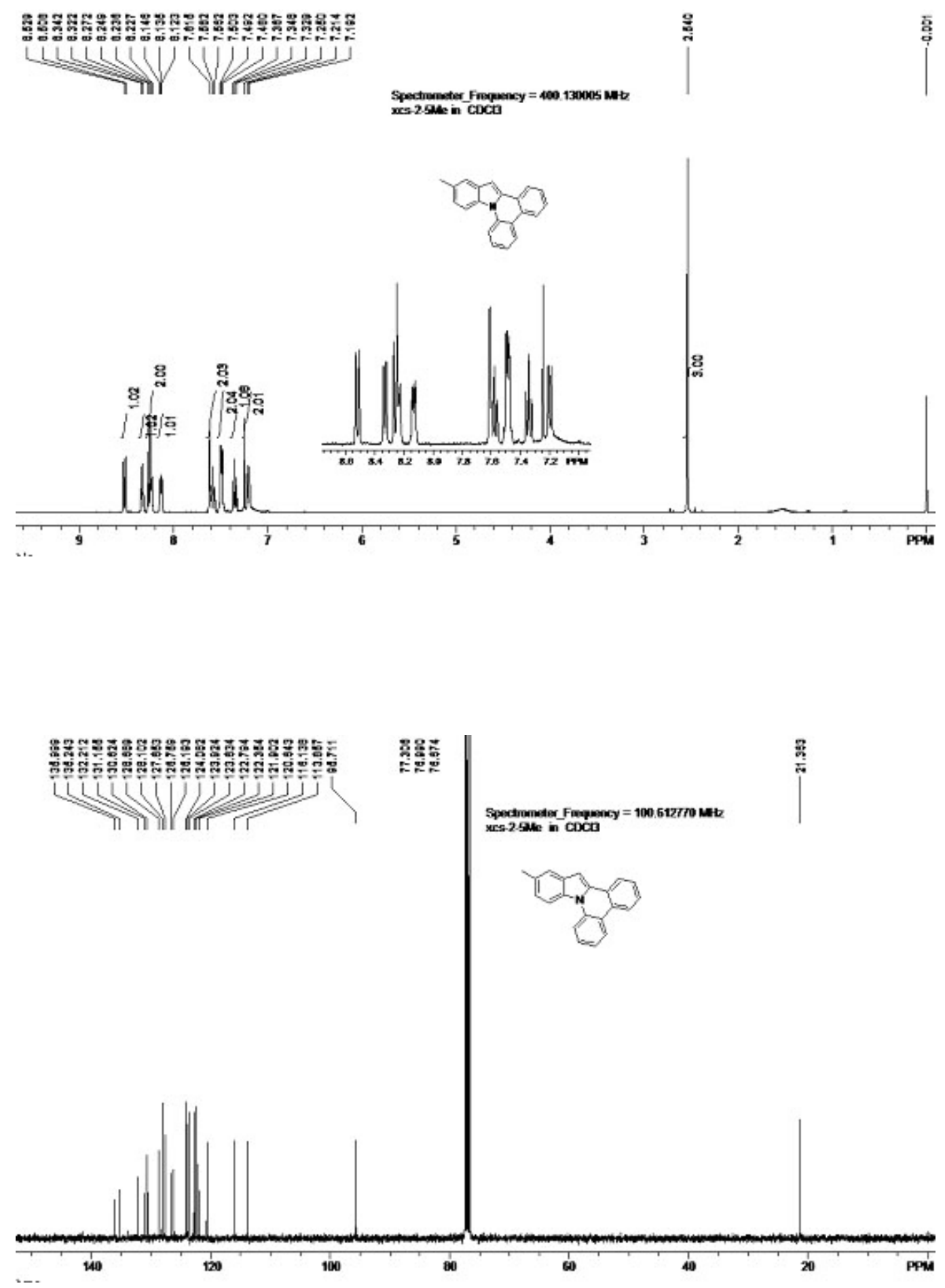
(4) 10-methylindolo[1,2-f]phenanthridine [ T 2-4, New compound ]

${ }^{1} \mathrm{H}$ NMR ( $400 \mathrm{MHz}, \mathrm{CDCl}_{3}$, TMS ) $\delta 8.57$ ( d, J=8.4 Hz, $1 \mathrm{H}$ ), 8.35 ( d, $J=8 \mathrm{~Hz}, 1 \mathrm{H}$ ), 8.26 $(\mathrm{m}, 2 \mathrm{H}), 8.20(\mathrm{~m}, 1 \mathrm{H}), 7.60(\mathrm{t}, J=7.4 \mathrm{~Hz}, 1 \mathrm{H}), 7.51(\mathrm{t}, J=3.8 \mathrm{~Hz}, 2 \mathrm{H}), 7.37(\mathrm{t}, J=7.6$ $\mathrm{Hz}, 1 \mathrm{H}), 7.31$ ( t, $J=7.8 \mathrm{~Hz}, 1 \mathrm{H}$ ), $7.26(\mathrm{~s}, 1 \mathrm{H}), 7.17$ ( d, $J=6.8 \mathrm{~Hz}, 1 \mathrm{H}$ ), $2.72(\mathrm{~s}, 3 \mathrm{H}$ ); ${ }^{13} \mathrm{C}$ NMR ( $100 \mathrm{MHz}, \mathrm{CDCl}_{3}$ ) $\delta 136.0,134.6,133.6,130.2$, 130.0, 128.7, 128.1, 127.6, 126.7, 126.2, 124.0, 123.9, 123.0, 122.4, 122.1, 121.9, 119.5, 116.3, 111.8, 94.6, 19.0. HRMS (ESI) Calcd for $\mathrm{C}_{21} \mathrm{H}_{15} \mathrm{~N}$ : $[\mathrm{M}+\mathrm{H}]^{+}$282.1277; Found, 282.1273. Yellow powder, mp 192-193 ${ }^{\circ} \mathrm{C}$.

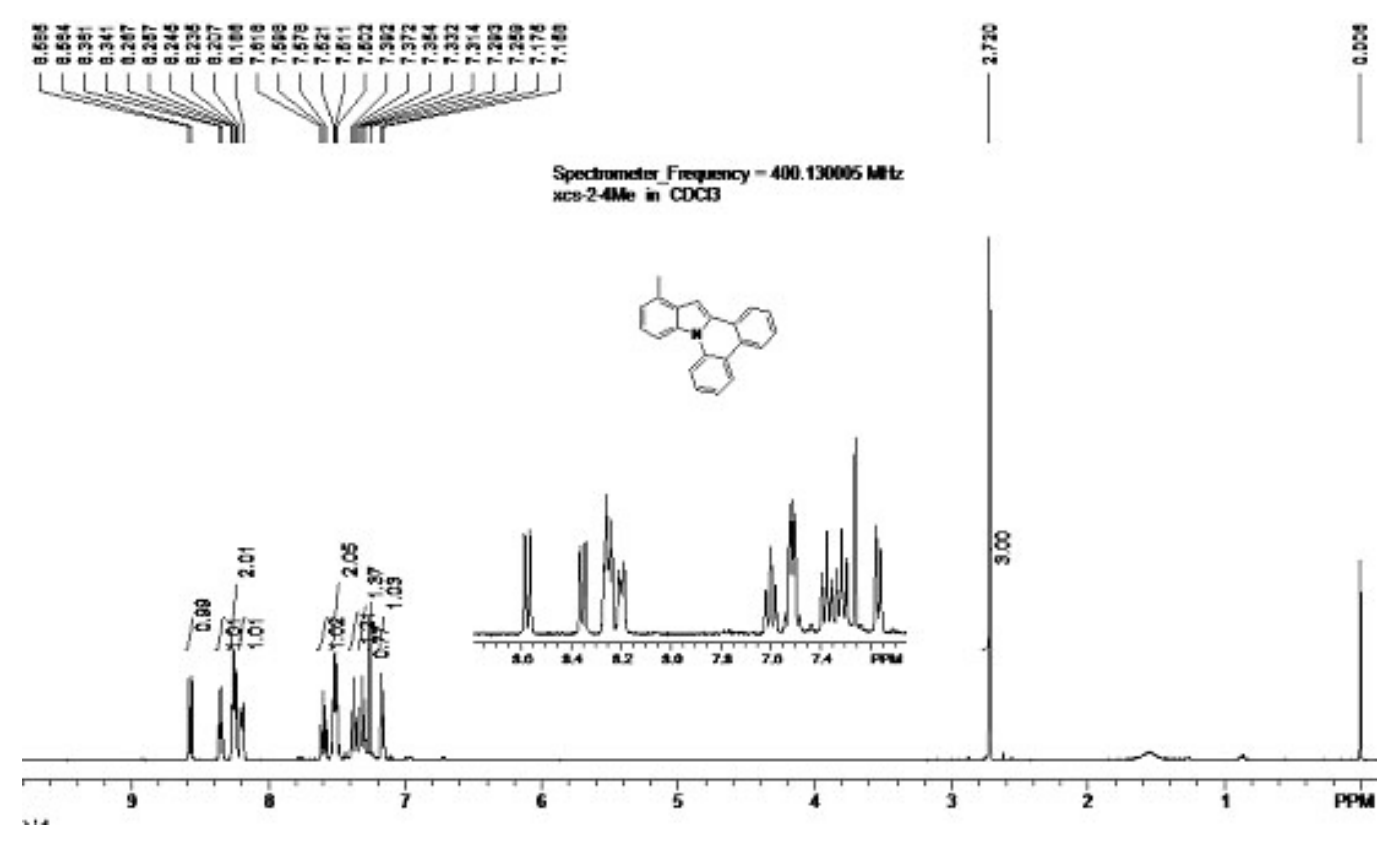




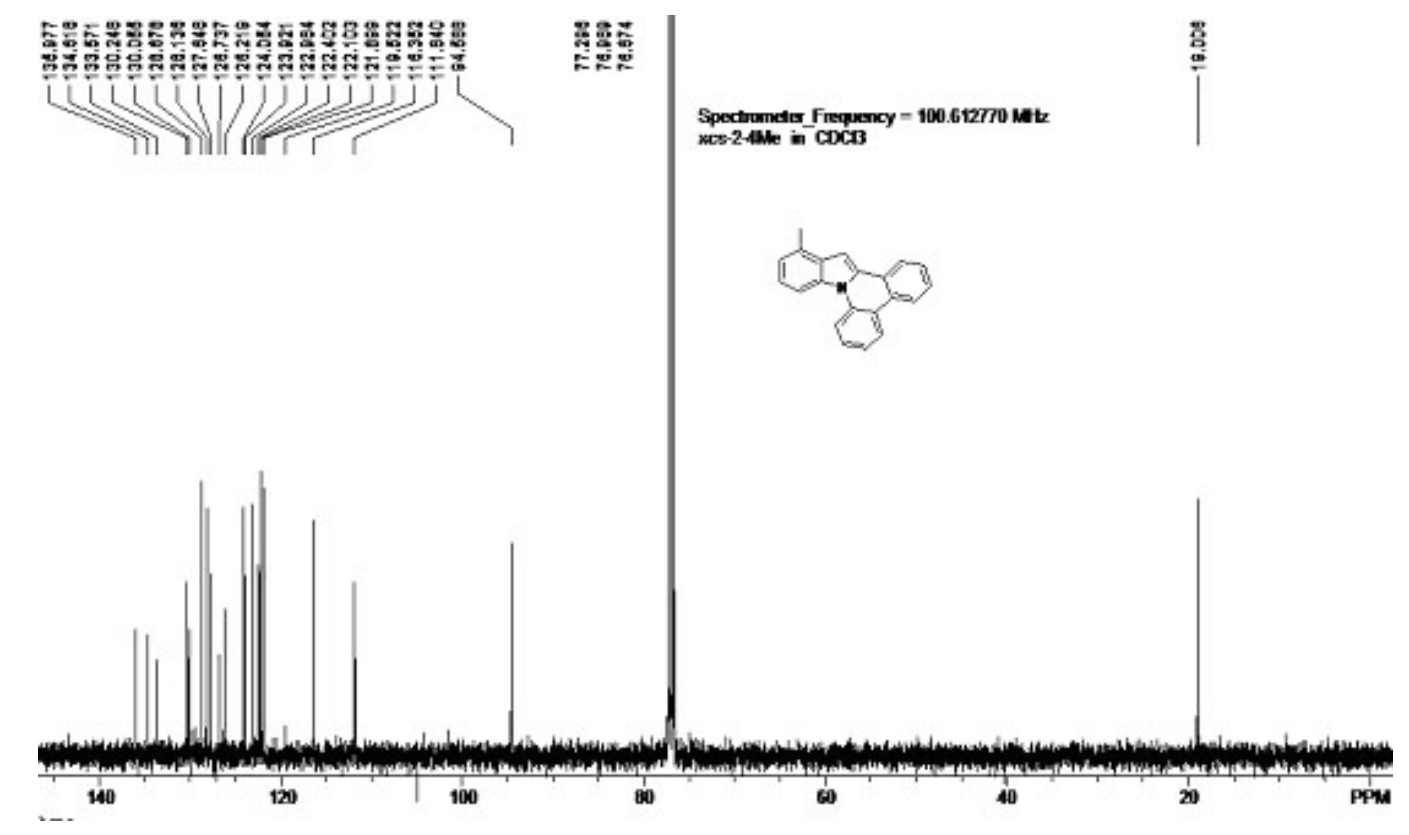

(5) indolo[1,2-f]phenanthridine [ T 2-5, New compound ]

${ }^{1} \mathrm{H}$ NMR ( $400 \mathrm{MHz}, \mathrm{CDCl}_{3}$, TMS ) $\delta 8.56(\mathrm{~d}, J=8.4 \mathrm{~Hz}, 1 \mathrm{H}$ ), $8.39(\mathrm{~d}, J=8.4 \mathrm{~Hz}, 1 \mathrm{H}$ ), $8.34(\mathrm{~d}, J=8 \mathrm{~Hz}, 1 \mathrm{H}), 8.24(\mathrm{~m}, 1 \mathrm{H}), 8.15(\mathrm{~m}, 1 \mathrm{H}), 7.84(\mathrm{~d}, J=7.6 \mathrm{~Hz}, 1 \mathrm{H}), 7.59(\mathrm{t}, J=$ $7.6 \mathrm{~Hz}, 1 \mathrm{H}$ ), $7.50(\mathrm{~m}, 2 \mathrm{H}), 7.36(\mathrm{~m}, 3 \mathrm{H}), 7.28$ ( s, $1 \mathrm{H}) ;{ }^{13} \mathrm{C} \mathrm{NMR}\left(100 \mathrm{MHz}, \mathrm{CDCl}_{3}\right) \delta$ 136.0, 135.2, 133.9, 130.3, 128.7, 128.2, 127.8, 126.8, 126.1, 124.1, 124.0, 123.0, 122.4, 122.1, 122.0, 121.8, 121.0, 116.3, 114.2, 96.2. HRMS (ESI) Calcd for $\mathrm{C}_{20} \mathrm{H}_{13} \mathrm{~N}$ : $[\mathrm{M}+\mathrm{H}]^{+}$268.1121; Found, 268.1113. Yellow powder, mp $140-142^{\circ} \mathrm{C}$. 

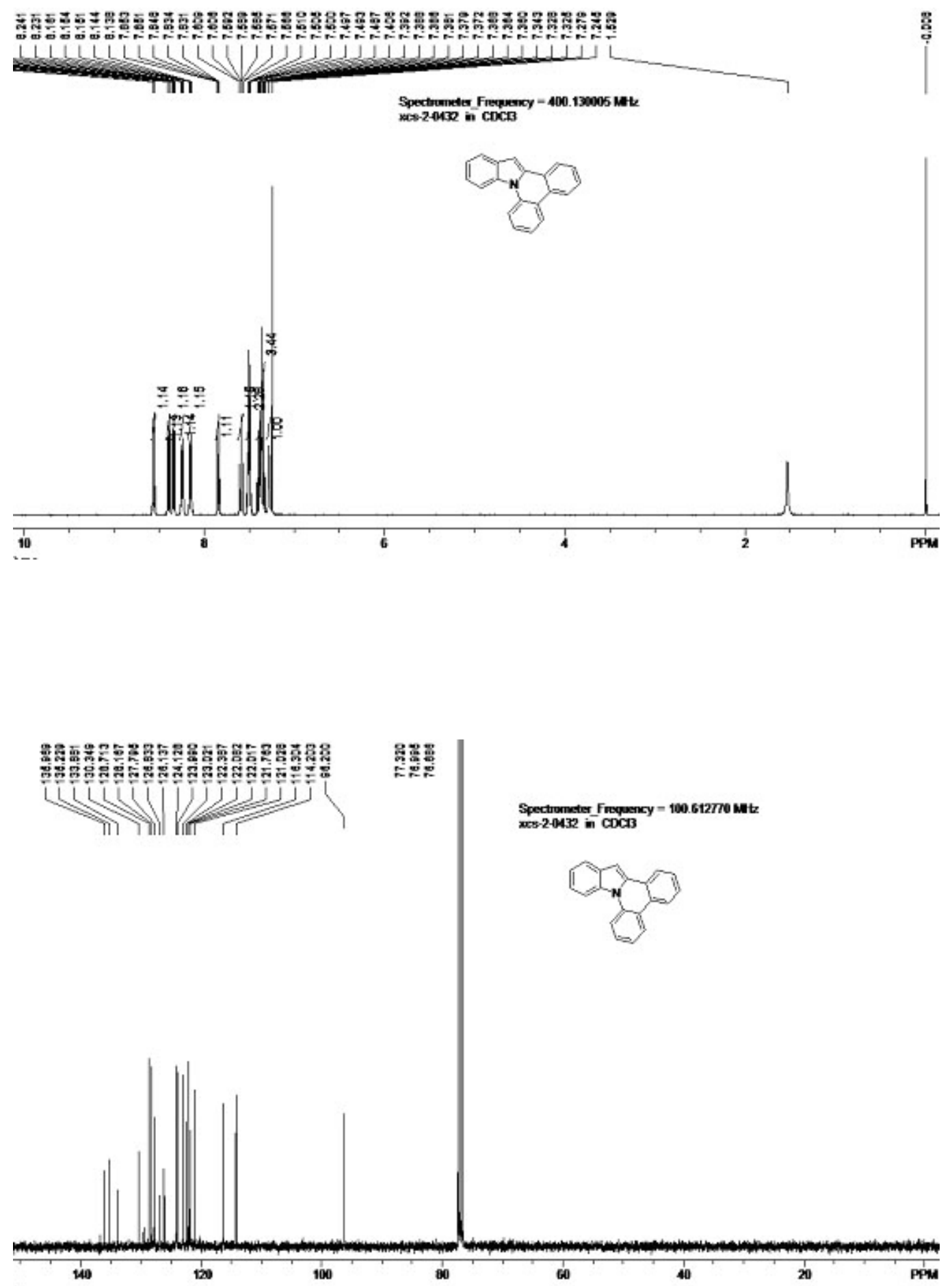
(6) methyl indolo[1,2-f]phenanthridine-10-carboxylate [ T 2-6, New compound ]

${ }^{1} \mathrm{H}$ NMR ( $400 \mathrm{MHz}, \mathrm{CDCl}_{3}$, TMS ) $\delta 8.59$ ( d, $J=8.4 \mathrm{~Hz}, 1 \mathrm{H}$ ), $8.51(\mathrm{~d}, J=8.4 \mathrm{~Hz}, 1 \mathrm{H}$ ), $8.37(\mathrm{~d}, J=8 \mathrm{~Hz}, 1 \mathrm{H}), 8.27(\mathrm{q}, 2 \mathrm{H}), 8.12(\mathrm{~d}, J=8 \mathrm{~Hz}, 1 \mathrm{H}), 8.02(\mathrm{~s}, 1 \mathrm{H}), 7.60(\mathrm{t}, J=7.6$ $\mathrm{Hz}, 1 \mathrm{H}$ ), $7.55(\mathrm{~m}, 2 \mathrm{H}), 7.40(\mathrm{t}, J=8.2 \mathrm{~Hz}, 2 \mathrm{H}), 4.06(\mathrm{~s}, 3 \mathrm{H}) ;{ }^{13} \mathrm{C} \mathrm{NMR}(100 \mathrm{MHz}$, $\left.\mathrm{CDCl}_{3}\right) \delta 167.6,136.9,135.0,134.5,129.8,128.5,128.1,128.0,126.8,125.4,124.8,124.4$, 123.8, 123.1, 122.0, 121.9, 120.6, 120.5, 118.3, 116.1, 97.0, 51.6. HRMS (ESI) Calcd for $\mathrm{C}_{22} \mathrm{H}_{15} \mathrm{NO}_{2}$ : $[\mathrm{M}+\mathrm{Na}]^{+}$348.0995; Found, 348.0994. Yellow powder, mp 149-151 ${ }^{\circ} \mathrm{C}$.

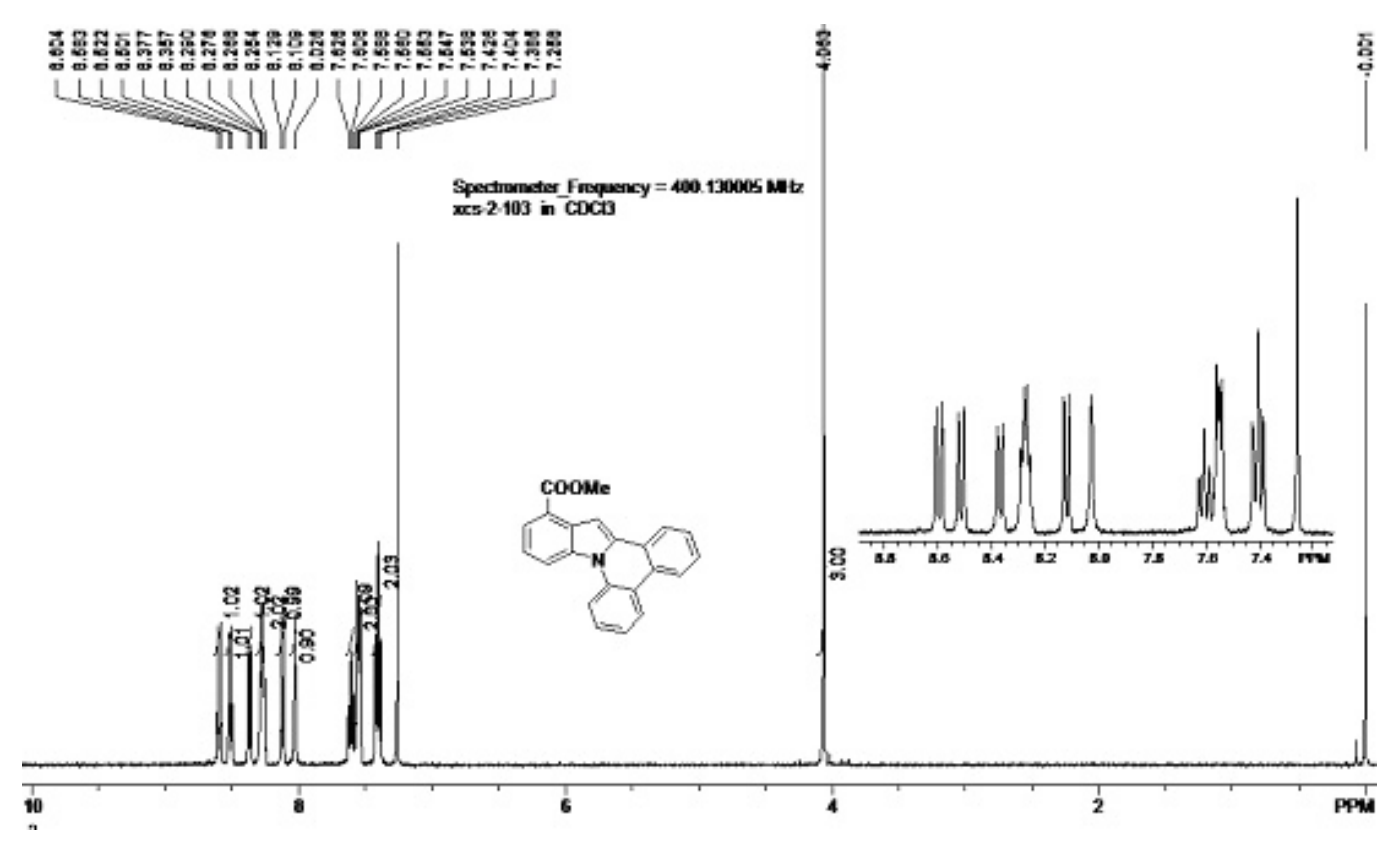




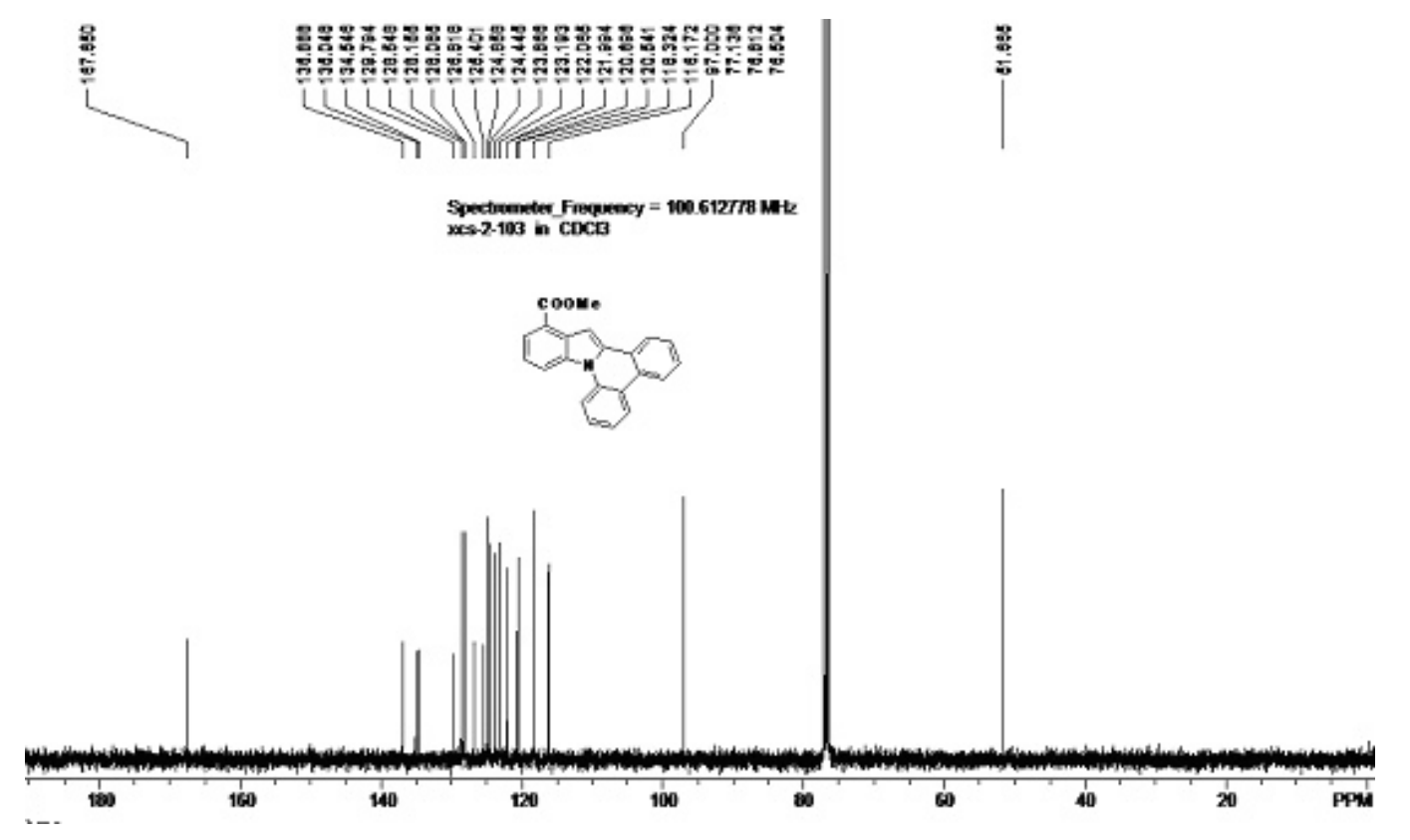

(7) indolo[1,2-f]phenanthridine-11-carbonitrile [ T 2-7, New compound ]

${ }^{1} \mathrm{H}$ NMR ( $400 \mathrm{MHz}, \mathrm{CDCl}_{3}$, TMS ) $\delta 8.32(\mathrm{~m}, 3 \mathrm{H}$ ), $8.20(\mathrm{~d}, J=7.2 \mathrm{~Hz}, 1 \mathrm{H}), 8.04(\mathrm{~s}, 2 \mathrm{H})$, 7.54 ( m, $4 \mathrm{H}$ ), 7.40 ( t $J=7.8 \mathrm{~Hz}, 1 \mathrm{H}$ ), $7.16(\mathrm{~s}, 1 \mathrm{H}) ;{ }^{13} \mathrm{C} \mathrm{NMR}\left(100 \mathrm{MHz}, \mathrm{CDCl}_{3}\right) \delta 137.1$, $134.89,134.86,129.8,128.9,128.7,128.5,126.9,125.9,125.1,124.3,124.2,124.1,122.4$, 122.3, 121.7, 120.1, 116.4, 114.6, 104.7, 96.2, 21.4. HRMS (ESI) Calcd for $\mathrm{C}_{21} \mathrm{H}_{12} \mathrm{~N}_{2}$ : $[\mathrm{M}+\mathrm{H}]^{+}$ 293.1073; Found, 293.1069. Yellow powder, mp 228-230 ${ }^{\circ} \mathrm{C}$. 

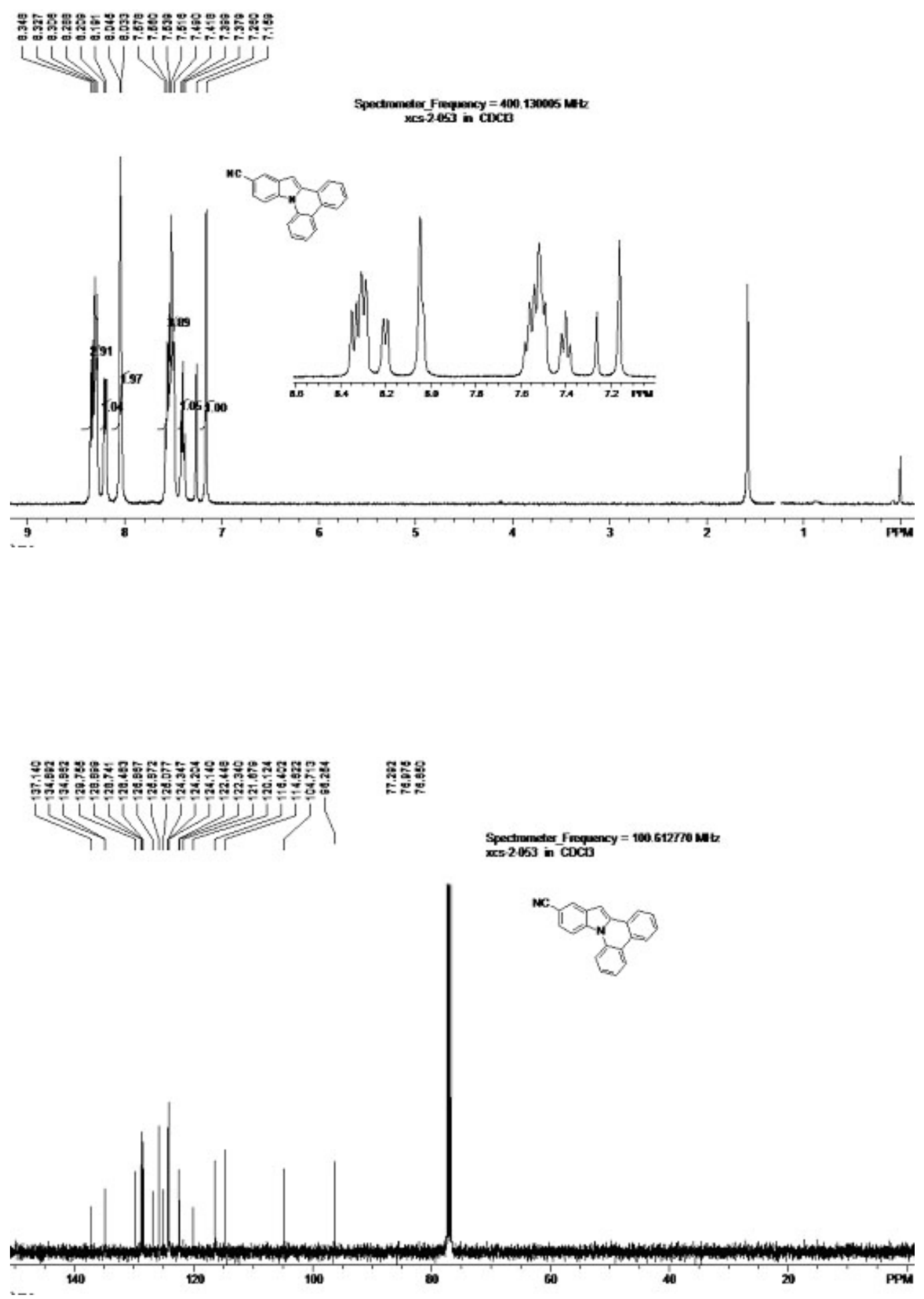
(8) 11-methoxy-6,7-dimethylindolo[1,2-f]phenanthridine [ T 2-8, New compound ]

${ }^{1} \mathrm{H}$ NMR ( $400 \mathrm{MHz}, \mathrm{CDCl}_{3}$, TMS ) $\delta 8.48(\mathrm{~d}, J=8.4 \mathrm{~Hz}, 1 \mathrm{H}$ ), $8.33(\mathrm{~d}, J=8 \mathrm{~Hz}, 1 \mathrm{H}$ ), 8.30 $(\mathrm{d}, \mathrm{J}=18 \mathrm{~Hz}, 1 \mathrm{H}$ ), $7.99(\mathrm{~s}, 1 \mathrm{H}), 7.88(\mathrm{~s}, 1 \mathrm{H}$ ), $7.56(\mathrm{t}, J=8 \mathrm{~Hz}, 1 \mathrm{H}), 7.34$ ( t,$J=7.4 \mathrm{~Hz}$, $1 \mathrm{H}), 7.27(\mathrm{~s}, 1 \mathrm{H}), 7.25(\mathrm{~s}, 1 \mathrm{H}), 7.01(\mathrm{~d}, \mathrm{~J}=8.8 \mathrm{~Hz}, 1 \mathrm{H}), 3.95(\mathrm{~s}, 3 \mathrm{H}), 2.44(\mathrm{~s}, 3 \mathrm{H})$, 2.43 ( s, $3 \mathrm{H}$ ); ${ }^{13} \mathrm{C}$ NMR ( $100 \mathrm{MHz}, \mathrm{CDCl}_{3}$ ) $\delta$ 154.9, 137.0, 136.6, 135.9, 135.4, 131.4, 128.9, $127.9,124.5,124.4,123.6,123.5,122.8,122.4,121.8,115.6,114.9,111.2,101.9,94.8,55.5$, 20.2, 19.8. HRMS (ESI) Calcd for $\mathrm{C}_{23} \mathrm{H}_{19} \mathrm{NO}$ : $[\mathrm{M}+\mathrm{H}]^{+}$326.1539; Found, 326.1532. Yellow powder, mp $143-145^{\circ} \mathrm{C}$.

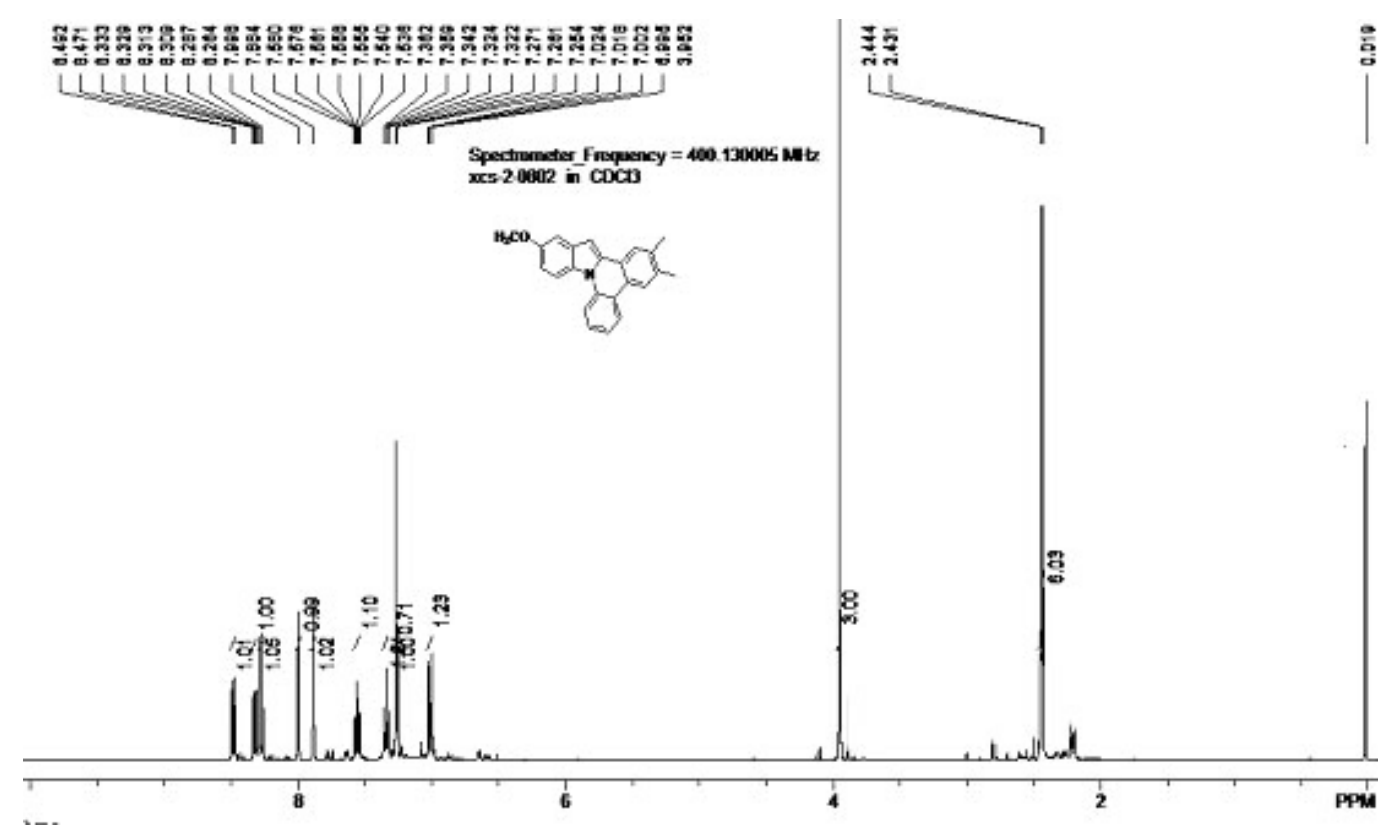




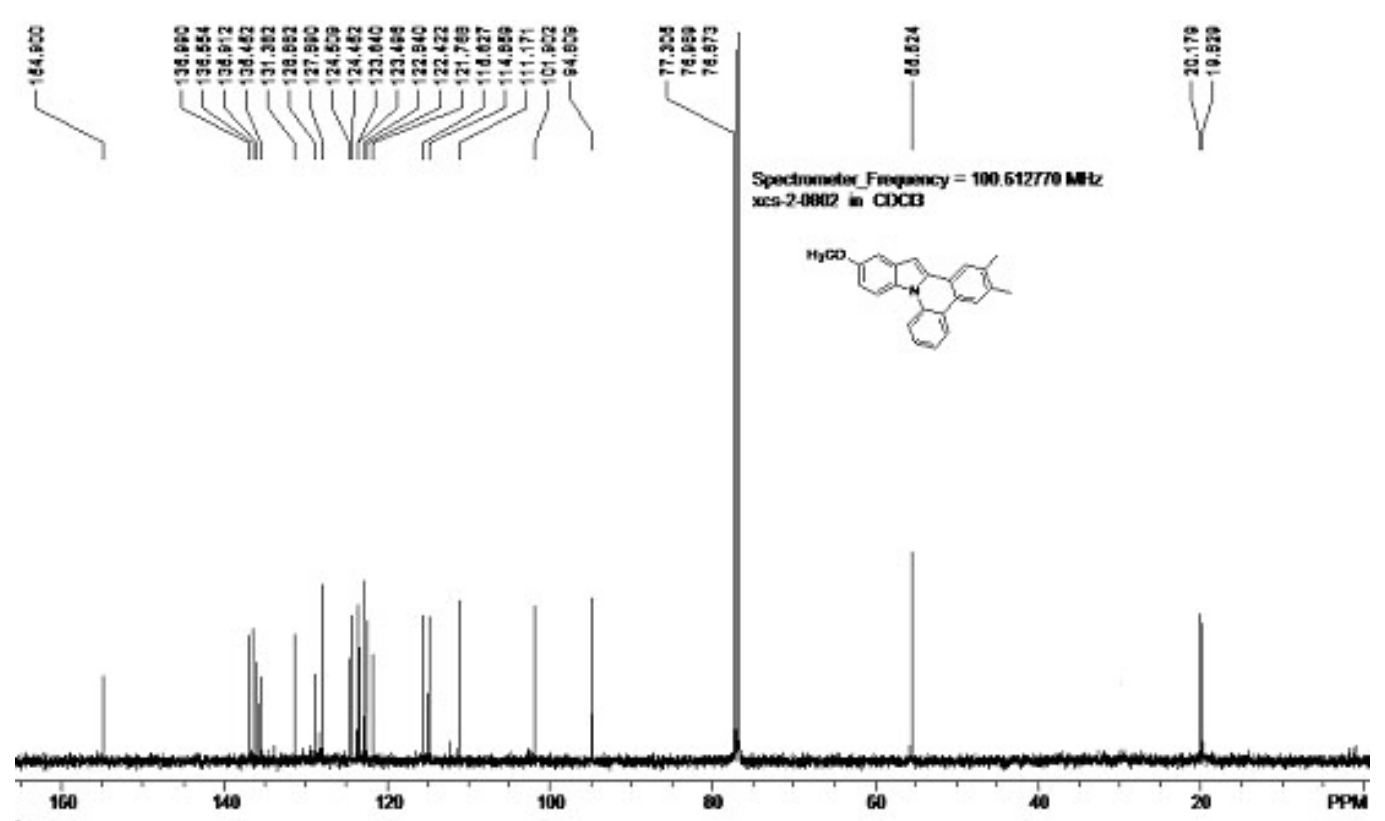

(9) 12-methoxy-6,7-dimethylindolo[1,2-f]phenanthridine [ T 2-9, New compound ]

${ }^{1} \mathrm{H}$ NMR ( $400 \mathrm{MHz}, \mathrm{CDCl}_{3}$, TMS ) $\delta 8.36(\mathrm{~d}, J=8.4 \mathrm{~Hz}, 1 \mathrm{H}$ ), $8.17(\mathrm{~d}, J=8 \mathrm{~Hz}, 1 \mathrm{H}$ ), 7.84 ( s, $1 \mathrm{H}), 7.81(\mathrm{~s}, 1 \mathrm{H}), 7.70(\mathrm{~s}, 1 \mathrm{H}), 7.69(\mathrm{~d}, J=8.8 \mathrm{~Hz}, 1 \mathrm{H}), 7.48(\mathrm{t}, J=7.8 \mathrm{~Hz}, 1 \mathrm{H})$, $7.45(\mathrm{~d}, \mathrm{~J}=4.4 \mathrm{~Hz}, 1 \mathrm{H}), 7.27(\mathrm{t}, J=7.4 \mathrm{~Hz}, 1 \mathrm{H}), 7.05(\mathrm{t}, J=9.2 \mathrm{~Hz}, 1 \mathrm{H}), 7.03(\mathrm{~s}, 1 \mathrm{H})$, 4.00 ( s, $3 \mathrm{H}$ ), 2.34 ( s, $3 \mathrm{H}$ ), 2.33 ( s, $3 \mathrm{H}$ ); ${ }^{13} \mathrm{C} \mathrm{NMR}\left(100 \mathrm{MHz}, \mathrm{CDCl}_{3}\right) \delta 155.8,137.1$, $135.5,133.9,129.6,129.3,127.7,124.8,124.24,124.18,124.1,123.5,122.8,122.7,122.3$, 121.1, 115.7, 110.4, 99.2, 94.1, 56.1, 20.1, 19.8. HRMS (ESI) Calcd for $\mathrm{C}_{23} \mathrm{H}_{19} \mathrm{NO}:[\mathrm{M}+\mathrm{H}]^{+}$ 326.1539; Found, 326.1532. Yellow powder, $\mathrm{mp} 89-91^{\circ} \mathrm{C}$. 

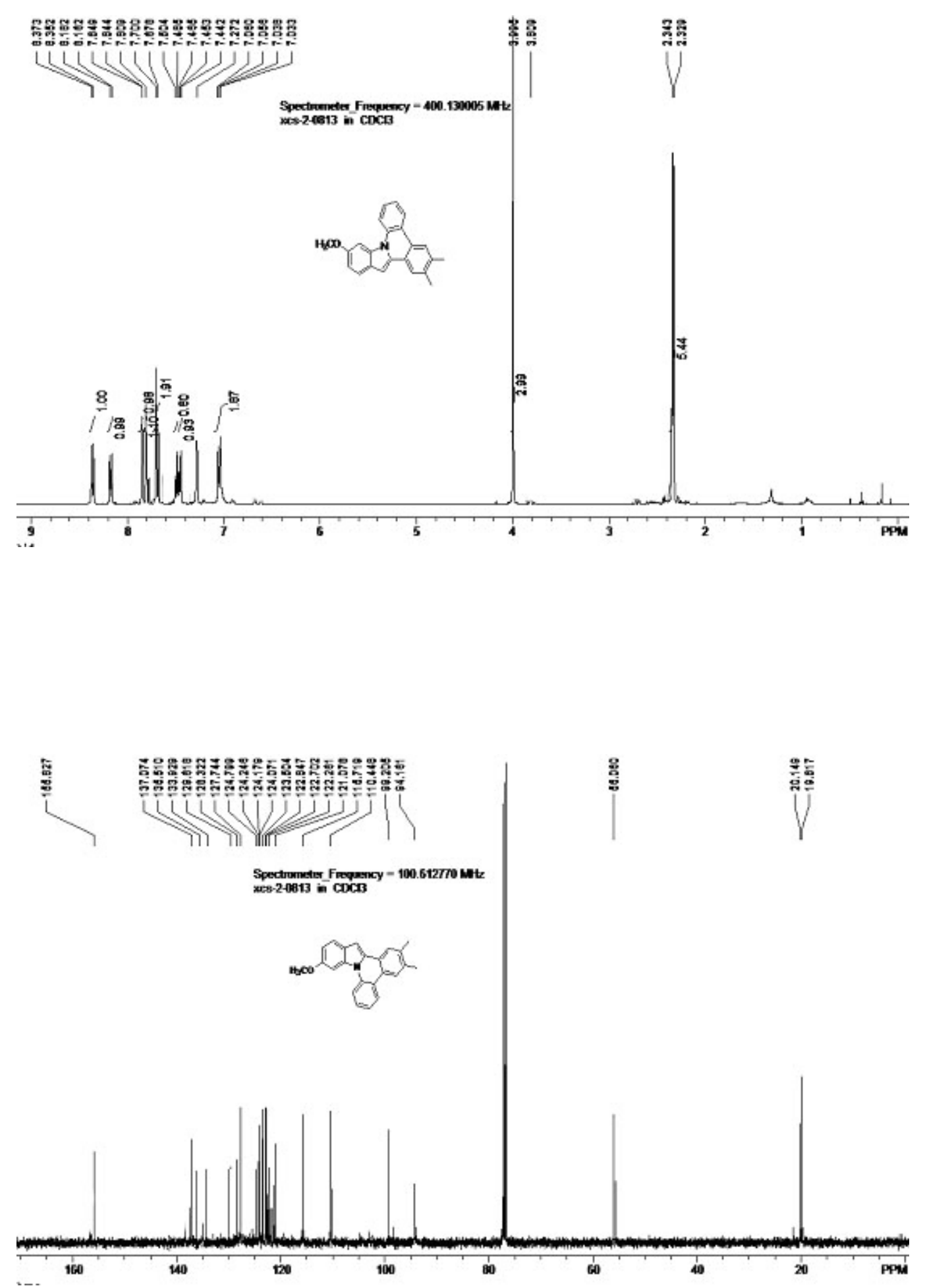
(10) 6,7,11-trimethylindolo[1,2-f]phenanthridine [ T 2-10, New compound ]

${ }^{1} \mathrm{H}$ NMR ( $400 \mathrm{MHz}, \mathrm{CDCl}_{3}$, TMS ) $\delta 8.51(\mathrm{~d}, J=8.4 \mathrm{~Hz}, 1 \mathrm{H}$ ), $8.30(\mathrm{~d}, J=8 \mathrm{~Hz}, 1 \mathrm{H}$ ), 8.27 $(\mathrm{d}, \mathrm{J}=8.4 \mathrm{~Hz}, 1 \mathrm{H}), 7.97(\mathrm{~s}, 1 \mathrm{H}), 7.87(\mathrm{~s}, 1 \mathrm{H}), 7.62(\mathrm{~s}, 1 \mathrm{H}), 7.56(\mathrm{t}, J=7.8 \mathrm{~Hz}, 1 \mathrm{H})$, $7.34(\mathrm{t}, J=7.8 \mathrm{~Hz}, 1 \mathrm{H}$ ), $7.28(\mathrm{~s}, 1 \mathrm{H}$ ), 7.20 ( d, J = 8.4 Hz, $1 \mathrm{H}$ ), 2.57 ( s, $3 \mathrm{H}$ ), 2.43 ( s, 3 $\mathrm{H}), 2.42(\mathrm{~s}, 3 \mathrm{H}) ;{ }^{13} \mathrm{C}$ NMR $\left(100 \mathrm{MHz}, \mathrm{CDCl}_{3}\right) \delta 137.1,136.6,135.7,135.4,132.1,130.9$, $130.7,127.9,124.6,123.9,123.5,123.2,122.9,122.6,121.9,120.4,120.1,116.0,113.8,94.8$, 21.3, 20.2, 19.8. HRMS (ESI) Calcd for $\mathrm{C}_{23} \mathrm{H}_{19} \mathrm{~N}$ : $[\mathrm{M}+\mathrm{H}]^{+}$310.1590; Found, 310.1592. Yellow powder, mp $135-136^{\circ} \mathrm{C}$.

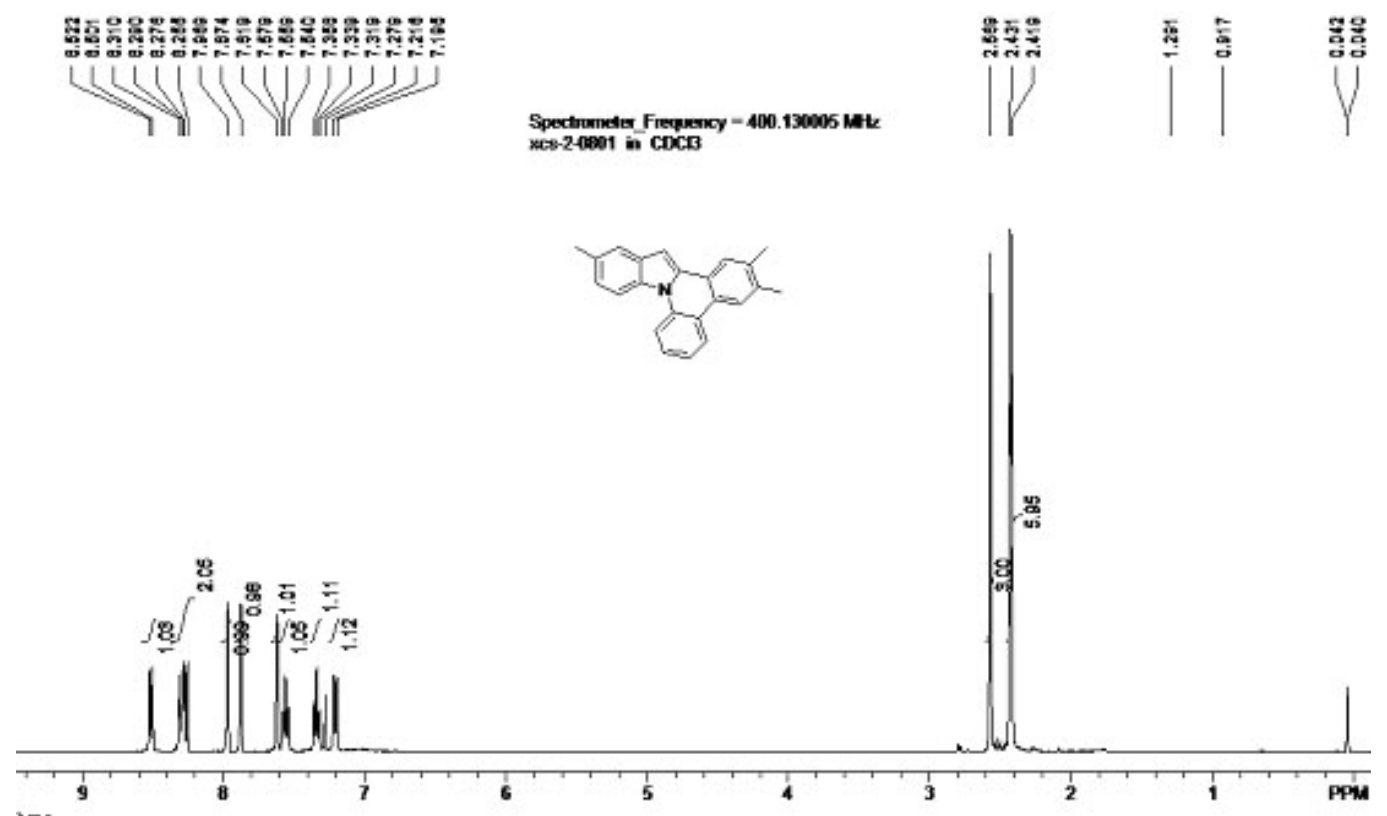




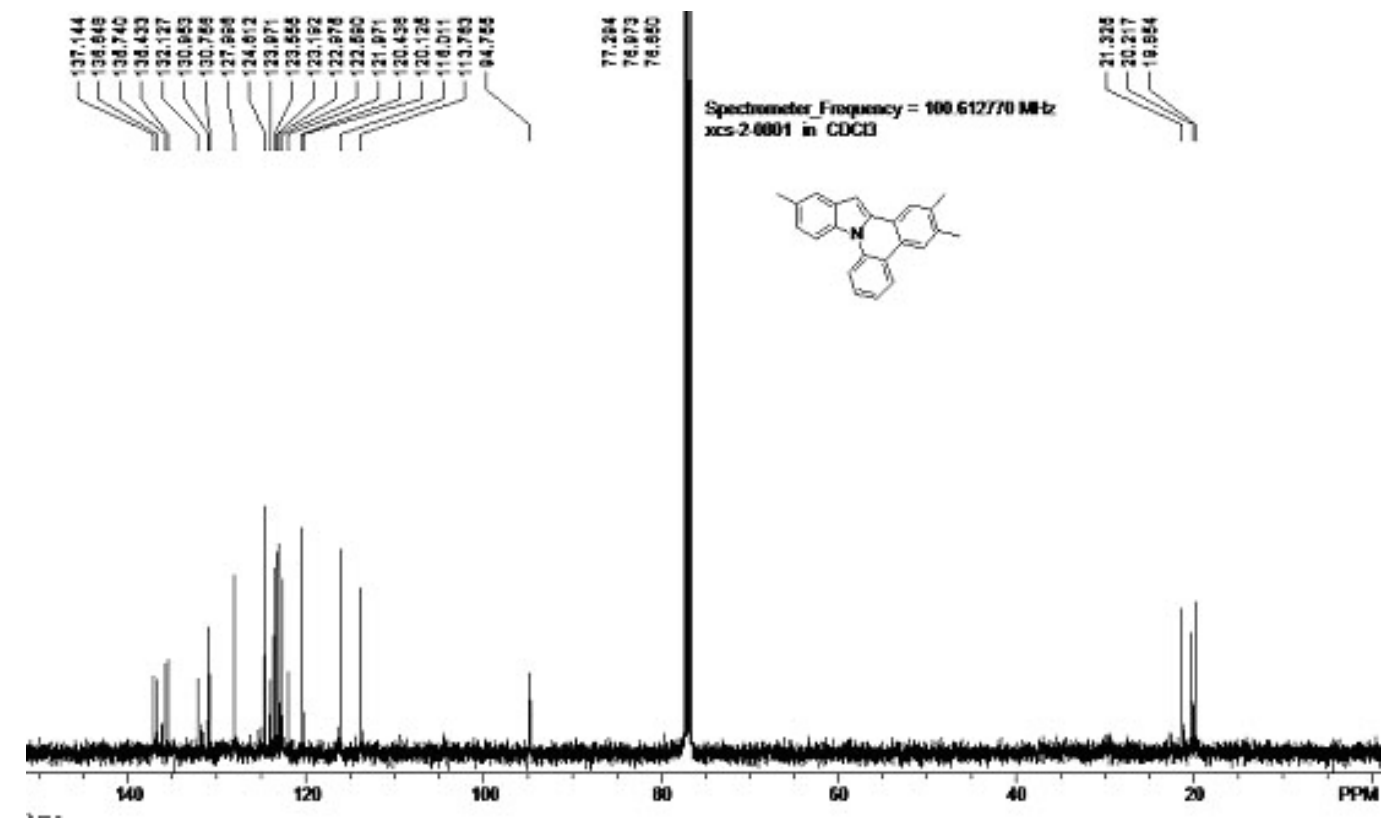

(11) 6,7,10-trimethylindolo[1,2-f]phenanthridine[ T 2-11, New compound ]

${ }^{1} \mathrm{H}$ NMR ( $400 \mathrm{MHz}, \mathrm{CDCl}_{3}$, TMS ) $\delta 8.50(\mathrm{~d}, J=8.4 \mathrm{~Hz}, 1 \mathrm{H}), 8.21(\mathrm{~d}, J=8.4 \mathrm{~Hz}, 1 \mathrm{H})$, $7.86(\mathrm{~s}, 1 \mathrm{H}), 7.81(\mathrm{~s}, 1 \mathrm{H}), 7.52$ ( t $, J=7.8 \mathrm{~Hz}, 1 \mathrm{H}$ ), 7.30 ( t $, J=7.8 \mathrm{~Hz}, 1 \mathrm{H}$ ), 7.18 ( s, $1 \mathrm{H}$ ), $7.16(\mathrm{~s}, 1 \mathrm{H}), 2.73(\mathrm{~s}, 3 \mathrm{H}), 2.36(\mathrm{~s}, 3 \mathrm{H}), 2.35$ ( s, $3 \mathrm{H}) ;{ }^{13} \mathrm{C} \mathrm{NMR}\left(100 \mathrm{MHz}, \mathrm{CDCl}_{3}\right) \delta$ $137.1,136.6,135.7,134.9,133.4,130.1,129.9,127.9,124.53,124.49,123.9,123.5,122.9$, 122.7, 122.1, 121.7, 121.6, 116.1, 111.8, 93.3, 20.2, 19.8, 19.0. HRMS (ESI) Calcd for $\mathrm{C}_{23} \mathrm{H}_{19} \mathrm{~N}$ : $[\mathrm{M}+\mathrm{H}]^{+} 310.1590$; Found, 310.1592. Yellow powder, mp $123-124^{\circ} \mathrm{C}$. 


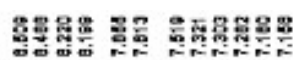

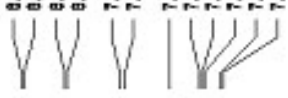

Spectrumeder Frequency $=400.130006 \mathrm{MeL}$

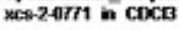

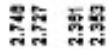

(1)
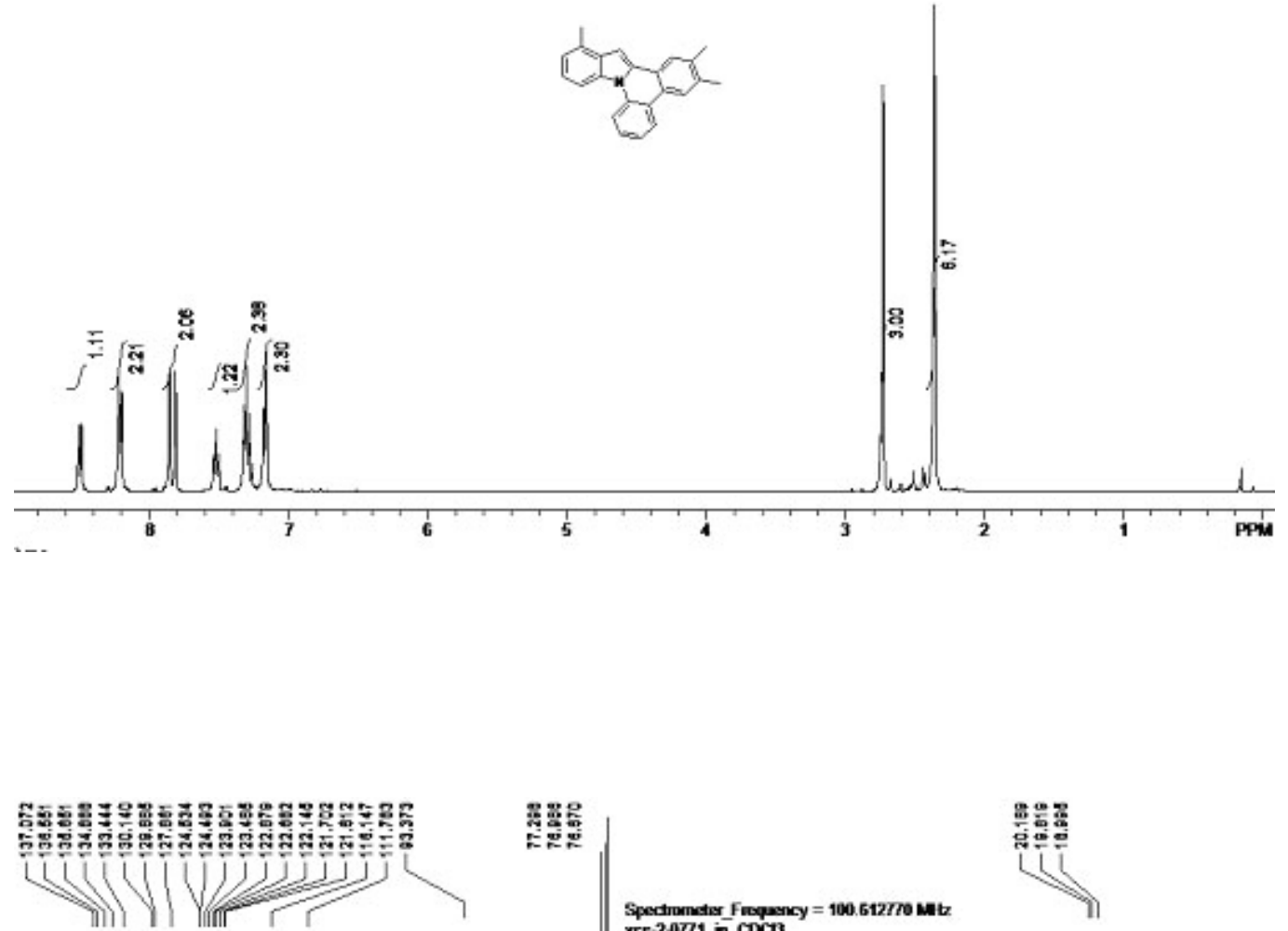

ב⿱艹

Spectrometis Frepaency $=100.6127 \mathrm{To}$ mat Spectrometis_ fropsents
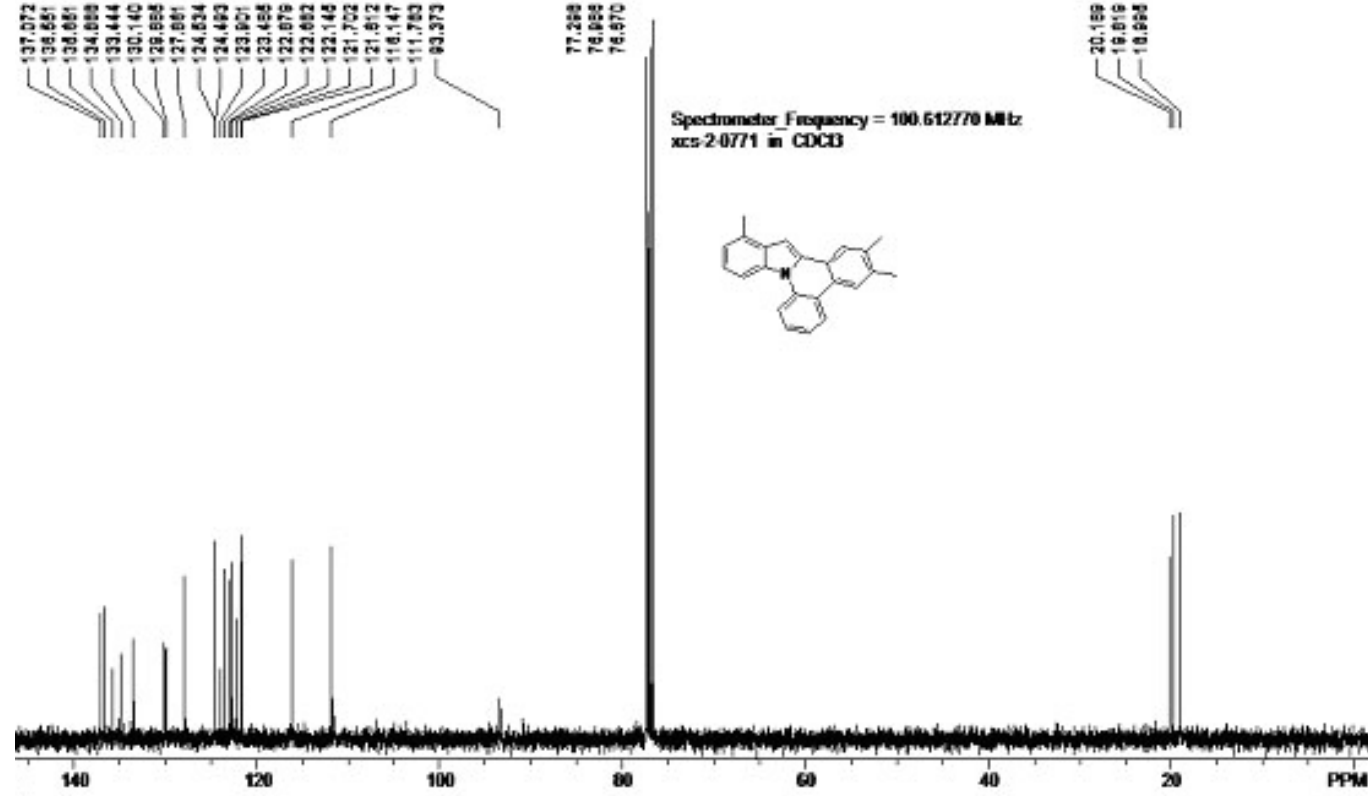
(12) 6,7-dimethylindolo[1,2-f]phenanthridine[ T 2-12, New compound ]

${ }^{1} \mathrm{H}$ NMR ( $400 \mathrm{MHz}, \mathrm{CDCl}_{3}$, TMS ) $\delta 8.51(\mathrm{~d}, J=8.4 \mathrm{~Hz}, 1 \mathrm{H}$ ), $8.38(\mathrm{~d}, J=6.8 \mathrm{~Hz}, 1 \mathrm{H}$ ), $8.24(\mathrm{~d}, J=8.0 \mathrm{~Hz}, 1 \mathrm{H}), 7.90(\mathrm{~s}, 1 \mathrm{H}), 7.82(\mathrm{~s}, 1 \mathrm{H}), 7.54(\mathrm{t}, J=8.0 \mathrm{~Hz}, 1 \mathrm{H}), 7.39$ ( m, 2 $\mathrm{H}), 7.32(\mathrm{t}, J=7.8 \mathrm{~Hz}, 1 \mathrm{H}), 7.17(\mathrm{~m}, 1 \mathrm{H}), 2.39(\mathrm{~s}, 3 \mathrm{H}), 2.37(\mathrm{~s}, 3 \mathrm{H}) ;{ }^{13} \mathrm{C} \mathrm{NMR}(100$ $\left.\mathrm{MHz}, \mathrm{CDCl}_{3}\right) \delta 137.1,136.7,135.6,135.4,133.7,130.4,127.9,124.62,124.59,123.8,123.6$, 122.9, 122.7, 122.1, 121.54, 121.52, 120.7, 116.1, 114.1, 95.1, 20.2, 19.8. HRMS (ESI) Calcd for $\mathrm{C}_{22} \mathrm{H}_{17} \mathrm{~N}$ : $[\mathrm{M}+\mathrm{H}]^{+} 296.1434$; Found, 296.1425. Yellow powder, mp 103-105 ${ }^{\circ} \mathrm{C}$.

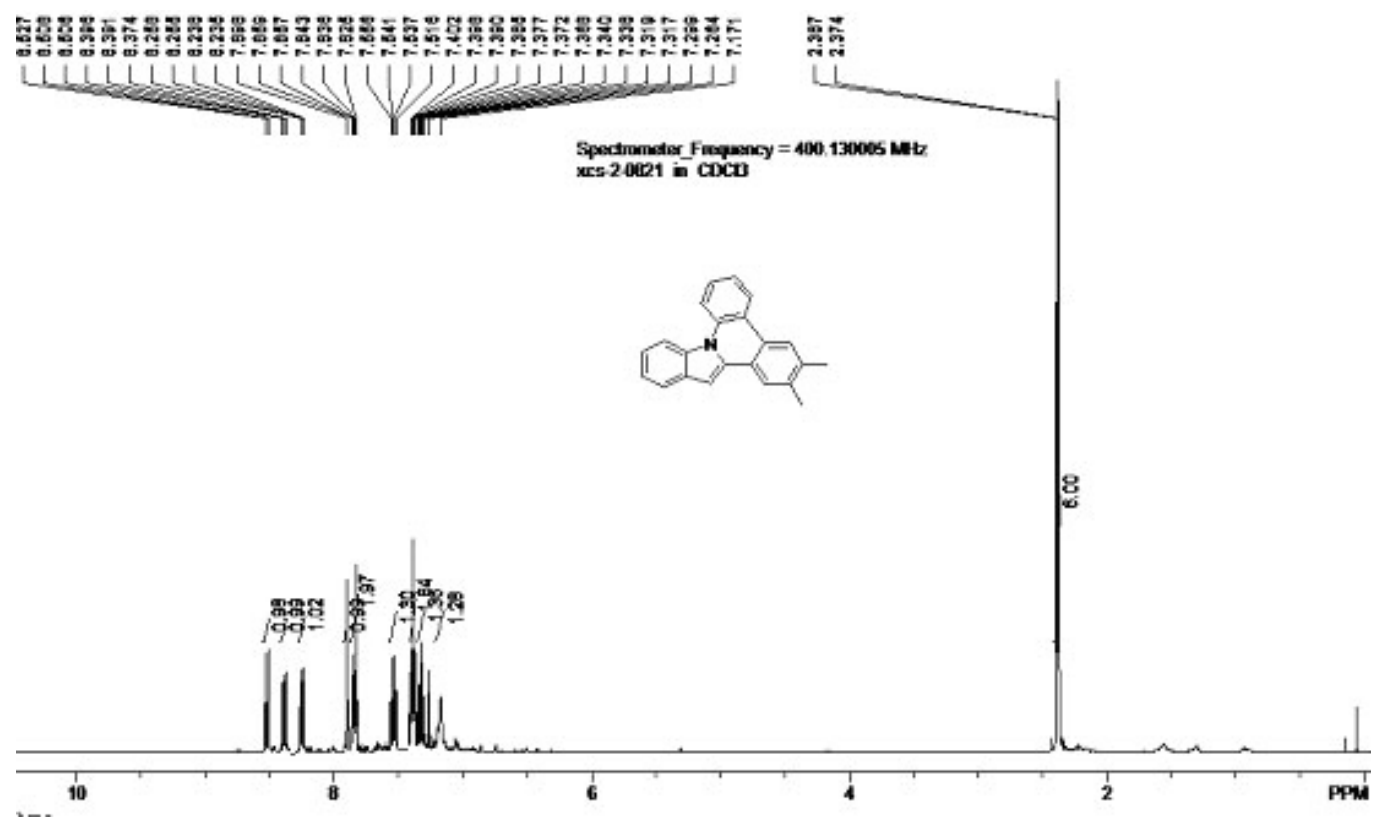




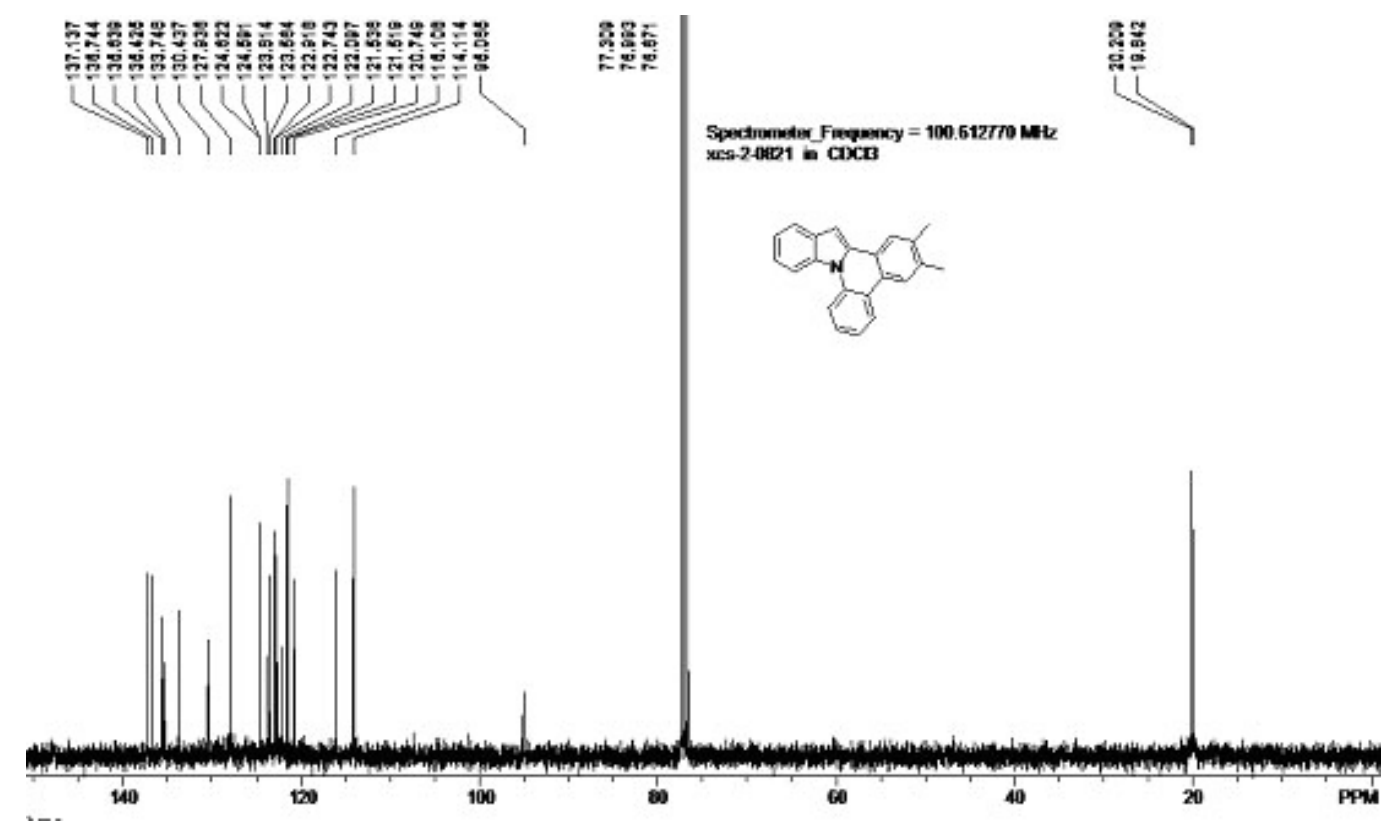

UV-Vis absorbance spectra and fluorescence spectra of the selected new compounds
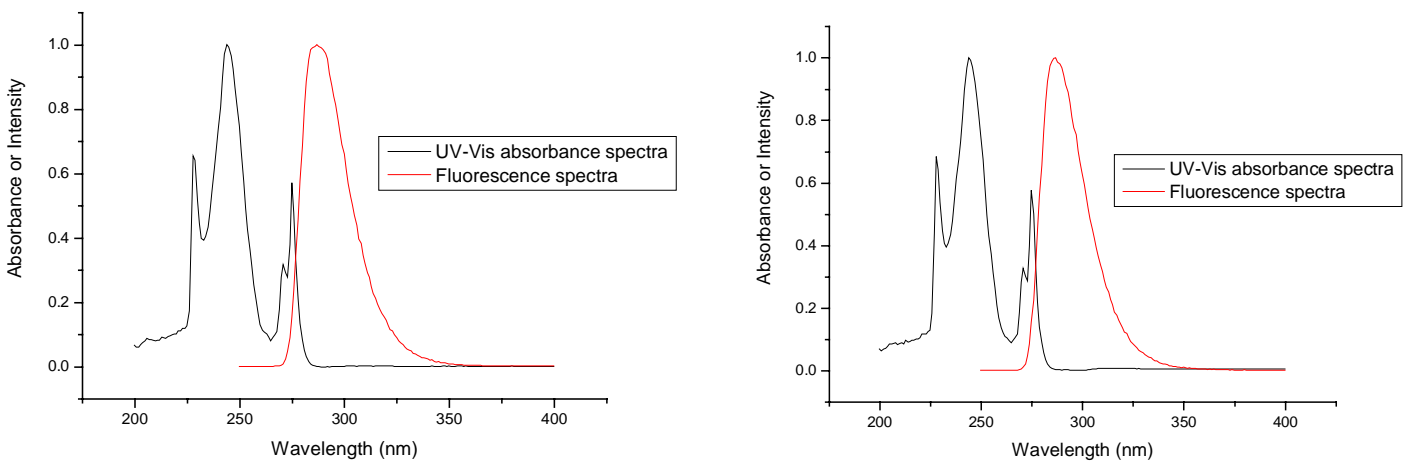

UV-Vis absorbance spectra and Fluoresence spectra of T 2-1 (Left) and T 2-2 (Right) 

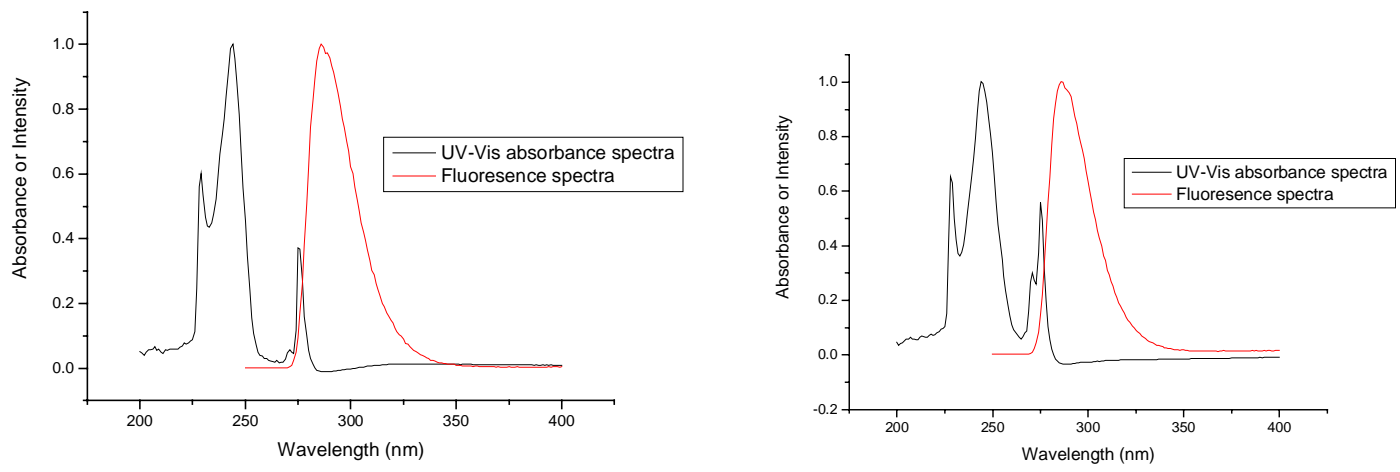

UV-Vis absorbance spectra and Fluoresence spectra of T 2-3 (Left) and T 2-4 (Right)

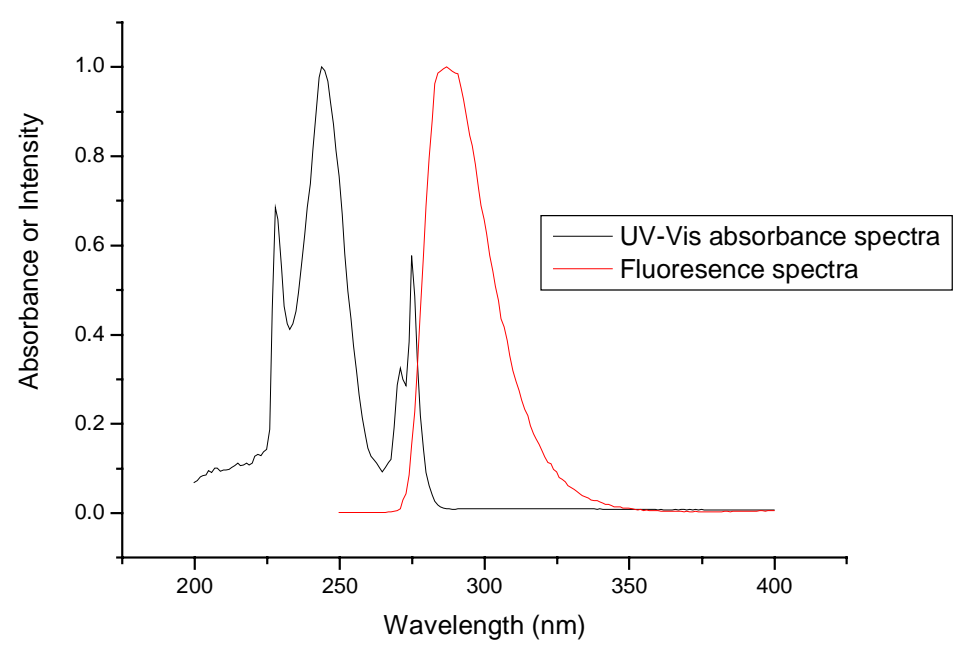

UV-Vis absorbance spectra and Fluoresence spectra of T 2-5 

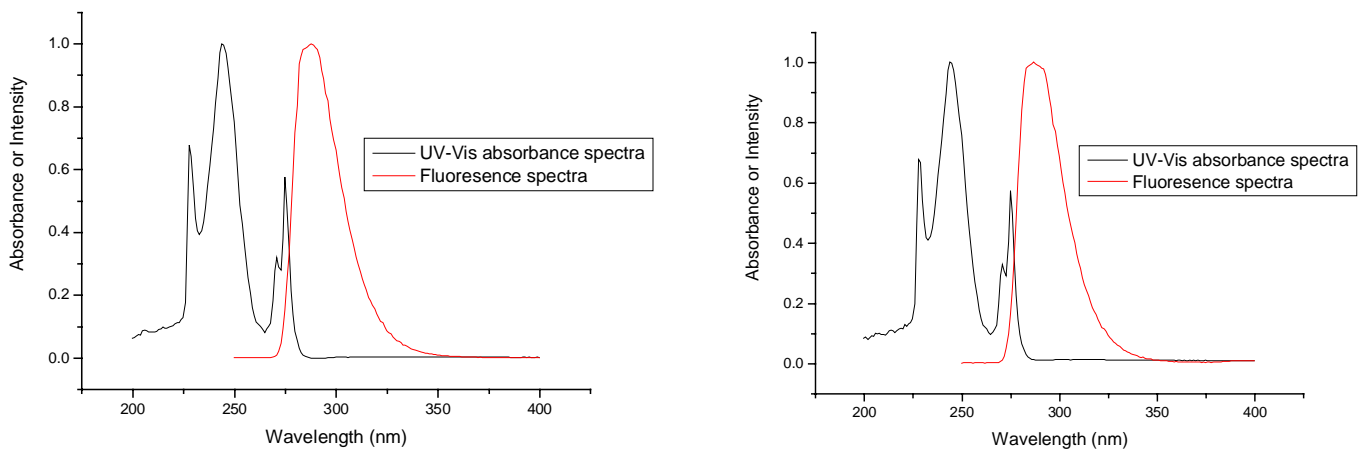

UV-Vis absorbance spectra and fluoresence spectra of T 2-6 (Left) and T 2-7 (Right)

Table 1 Fluorescence quantum yield of the above new compounds ${ }^{\text {a }}$

\begin{tabular}{|c|l|c|}
\hline entry & \multicolumn{1}{|c|}{$\mathrm{R}_{1}=$} & quantum yield $^{\mathrm{b}}$ \\
\hline 1 & $5-\mathrm{MeO}(\mathrm{T} 2-1)$ & 0.39 \\
\hline 2 & 6-MeO (T2-2) & 0.41 \\
\hline 3 & $5-\mathrm{Me}(\mathrm{T} 2-3)$ & 0.36 \\
\hline 4 & $4-\mathrm{Me}(\mathrm{T} 2-4)$ & 0.37 \\
\hline 5 & $\mathrm{H}(\mathrm{T} 2-5)$ & 0.28 \\
\hline 6 & 4-COOMe (T2-6) & 0.28 \\
\hline 7 & 5-CN (T2-7) & 0.27 \\
\hline
\end{tabular}

${ }^{\text {a }}$ Exciting wavelength was $275 \mathrm{~nm}$

${ }^{\mathrm{b}}$ Calculated in THF based on terphenyl as the standard $(\Phi=0.93,287 \mathrm{~nm})$ 
References

(1) Himeshima, Y.; Sonoda, T.; Kobayashi, H. Chem. Lett. 1983, 1211-1214.

(2) Klapars, A.; Antilla, J. C.; Huang, X. H.; Buchwald, S. L. J. Am. Chem. Soc. 2001, 123, 7727-7729. 